\title{
PID Control of Multisources Complex Excitations Active Vibration Isolation System: An Improved Particle Swarm Optimization Algorithm
}

\author{
Song Chunsheng, ${ }^{1,2}$ Jiang Youliang, ${ }^{1}$ and Zhang Jinguang ${ }^{1,2}$ \\ ${ }^{1}$ School of Mechanical and Electronic Engineering, Wuhan University of Technology, Wuhan 430070, China \\ ${ }^{2}$ Hubei Key Laboratory of Digital Manufacturing, Wuhan University of Technology, Wuhan 430070, China \\ Correspondence should be addressed to Song Chunsheng; song_chsh@163.com
}

Received 18 July 2016; Revised 25 September 2016; Accepted 19 October 2016

Academic Editor: Carlo Trigona

Copyright ( $\odot 2016$ Song Chunsheng et al. This is an open access article distributed under the Creative Commons Attribution License, which permits unrestricted use, distribution, and reproduction in any medium, provided the original work is properly cited.

\begin{abstract}
Active vibration isolation technology is the key technique to solve the vibration isolation problems related to the multisources complex excitations vibration isolation system. The electromagnetic actuators-based multisources complex excitations active vibration isolation system is built. Additionally, in view of the complex structure and strong coupling of the system, the least-squares method to identify and obtain the mathematical model of the vibration isolation system is adopted. Furthermore, this paper also sets up the acceleration feedback-based PID control model for multisources complex excitations active vibration isolation system, proposes an improved particle swarm optimization (PSO) algorithm of dynamic inertia weight factors used to optimize parameters of the built PID control model, and conducts simulation analysis. The simulation results show that, compared with the passive system before the control, the multisources complex excitations active vibration isolation system under the PID control has the far less peak-to-peak amplitude of acceleration which is transmitted to the foundation and has the much better vibration isolation effect. Finally, the paper conducts experimental verification, which demonstrates that active vibration control effect is identical to the simulation results and the vibration control effect is significantly improved.
\end{abstract}

\section{Introduction}

Along with the development of ships, the mechanical structure inside the ships becomes more and more complex, and so do the excitation mode and the components of excitation signals. Due to the limited load capacity and inner space of the ships, a majority of power devices are installed together rigidly or flexibly to form the isolation object with multiple excitation sources. As the vibration excitations generated by each equipment are different in amplitude, phase, frequency, and direction, those excitation signals are mixed and overlapped, resulting in complicated and variable frequency and phase of excitation signals which happen on the entire vibration isolation object [1-4]. The energy generated from mechanical vibration of the ships is mainly delivered via the supporting base. Vibration isolation technology can effectively isolate the mechanical vibration transfer. The vibration isolation technology can be divided into passive and active ones. Despite the simple structure and reliable operation, passive vibration isolation technology has inherent defect of poor vibration isolation performance in low frequency and resonance regions. It is difficult to purely rely on passive vibration isolation technology to further improve vibration isolation performance of multisources complex excitations vibration isolation system. Active vibration isolation technology can overcome the defect of the passive vibration isolation technology, which is the poor vibration isolation performance in low frequency and resonance regions. Thus, studies on theories, methods, and technologies of active vibration isolation technology under multiple complex excitation sources are critical to achieve vibration isolation of machinery equipment of ships in terms of whole frequency domain.

Active vibration isolation technology can be carried out from three aspects: structural optimization of the active 
vibration isolation system, control of active vibration isolation system, and active vibration isolator (actuator) [5]. The active noise and vibration control (ANVC) system was developed by the USA, which used marine high-speed network technology and was applied to full boat equipment for active vibration isolation below $100 \mathrm{~Hz}$ [6]. Four solenoid actuators were applied into single excitation active vibration isolation system, which effectively solved the control issue around the flexible plate with modal frequencies and obtained good vibration isolation performance [7]. A "smart spring" mounting system that ordinary springs and electric magnetic actuators were connected in parallel to was proposed. This system solved the response peak of traditional isolators and was applied to single excitation source active vibration isolation system $[8,9]$. A kind of vibration isolation structure was introduced, where the electrorheological fluid damper was used to change the damping force and adjust the system rigidity. This structure was applied to single excitation source active vibration isolation system and certain control algorithms were used, enabling the system rigidity to be adjustable within a certain range [10]. Active vibration isolation and system modeling of double-layer isolation system for ship engine or auxiliary engine with single excitation source was studied [11]. The literature [12] targeted the application of unidirectional excitation double-layer isolation system of electrorheological fluid damper to design a semiactive static output feedback fuzzy sliding mode controller. The simulation results showed that the vibration isolation performance was superior to that of the optimal passive damping system. The magnetic suspension technique was applied into microvibration platform with single excitation source and the theories of magnetic suspension vibration isolation under slight excitation were studied. Good vibration isolation effect was obtained [13]. Authorized by the US Navy, magnetic suspension vibration isolators were applied to floating raft isolation system, and active theories and control simulation experiments were studied, with good active vibration isolation effect $[14,15]$. Theoretical electromagnetic force model of the magnetic suspension vibration isolator with single excitation source according to measured data was modified [16].

Among the abovementioned research papers related to active vibration isolation, a majority focus on single-layer and double-layer active vibration isolation system with single excitation source, studying dynamics model and control strategy and algorithm, while some propose active control policies and guidelines of multifreedom degree vibration isolation system. However, there have yet been no deep and comprehensive studies about whether resultant effect can be achieved by jointly using multiple active vibration isolators. Multisources complex excitations active vibration isolation system is the system which achieves vibration isolation by giving play to combined effect of active and passive vibration isolation components. It is the typical mechatronic system. Due to the complicated and changeable excitation, strong nonlinear relationship, and interconnection among different excitation sources and different isolators via structure, the system is very complicated. Thus, it is difficult to build a mathematical model which meets the requirements with the analysis method. Experimental data include all information of the model and the use of model identification method in the experimental data is an effective way to solve such issues $[16,17]$. The least-squares method is the method which determines the parameter of system model by minimizing square and function of the generalized error. It is applicable to both linear and nonlinear systems. Thus, this paper adopts this method to complete model identification of multisources complex excitations active vibration isolation system. The control is the core link of the active vibration isolation system and the feedback control is especially suitable for complex systems and systems with uncertain parameters [17]. With simple structure, good stability, reliable operation, and strong robustness, PID feedback controller is mainly subject to three parameters, namely, P, I, and D. Determination of control parameters is the core of control system design. As taking of PID control parameters by hands not only is timeconsuming, but also fails to ensure optimal performance, intelligent control algorithm-based PID controller obtained the PID parameters by combining with modern intelligent algorithms such as genetic algorithm, ant colony optimization algorithms, and particle swarm optimization (PSO) algorithm [18-27]. The PSO algorithm is a kind of swarm optimization algorithm in the field of smart computing which is obtained by referring to preying of birds. Compared with the genetic algorithm, ant colony optimization algorithms, and other smart computing methods, the PSO algorithm has simple structure, few parameters, fast convergence speed, easiness to implement, and low time complexity and space complexity. It is demonstrated to obtain the good optimal solution at a low computational cost, and thus it is widely studied and applied in PID parameter optimization field [1927].

The response amplitude of the acceleration delivered to the foundation or the attenuation ratio in the course of acceleration passing is an important indicator to measure the vibration isolation effect of mechanical equipment. Therefore, taking the strong coupling, nonlinear characteristics of complex multisources excitation active vibration isolation system, and the difficulty in establishing an accurate model using traditional kinetic methods into account, the least-squares model identification method is adopted to establish the active vibration isolation system model under the multiple complex excitation sources and build the intelligent PID control model which is based on acceleration feedback. In addition, this paper proposes the improved PSO algorithm of dynamic inertia weight factor, optimizes the built PID control parameters, and conducts simulation analysis and finally experimental verification.

\section{Model of Multisources Complex Excitations Active Vibration Isolation System}

The model of multisources complex excitation active vibration isolation system is shown in Figure 1. Two vibration motors 1-1 and 1-2 are connected rigidly with plate 2 . By modulating the frequency via a convertor, the rational speed of two vibration motors can be adjusted and the amplitude and frequency of excitation signals can be changed and adjusted 

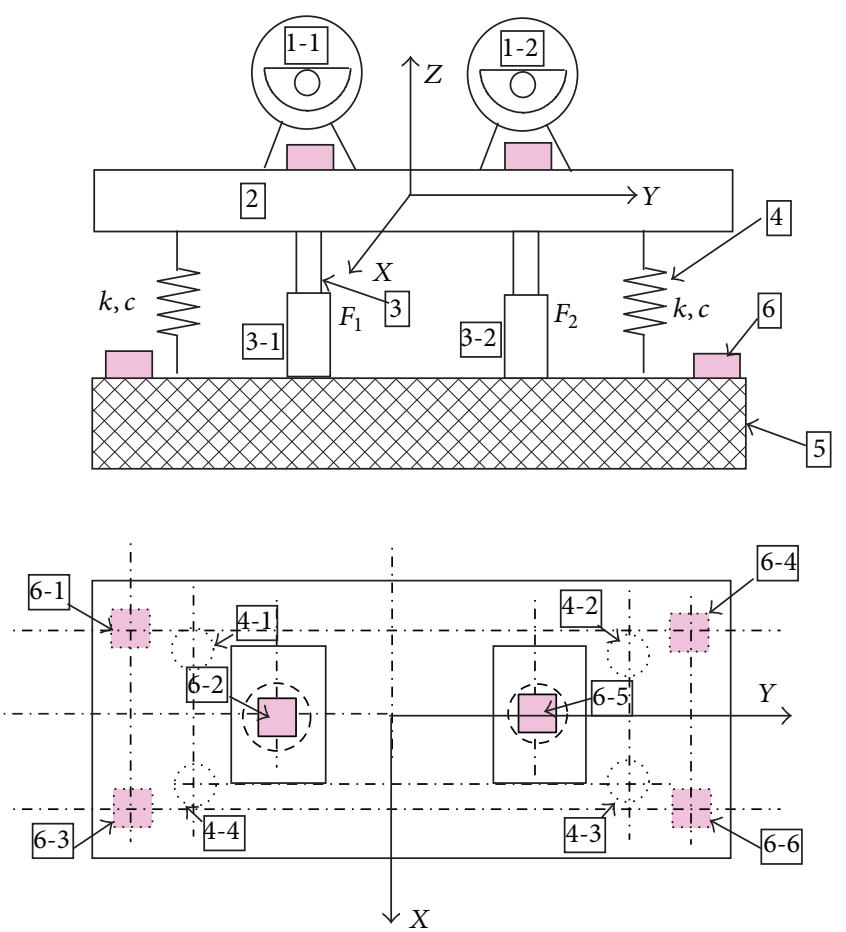

FIGURE 1: Multisources complex excitations active vibration isolation system.

upon the system need. Electromagnetic actuators 3-1 and 32 are installed between plate 2 and foundation 5 in parallel together with four rubber isolators 4-1, 4-2, 4-3, and 4-4. Six accelerometers 6-1, 6-2, 6-3, 6-4, 6-5, and 6-6 are used to measure the acceleration at the corresponding positions. $F_{1}$ and $F_{2}$ are electromagnetic forces of electromagnetic actuators 31 and 3-2. XYZ is the corresponding Cartesian coordinate system. Considering that the system targets minimizing the vibration transmitted to the foundation, the acceleration responses at four bottom positions of the vibration isolation system are chosen as the major indicators to evaluate the vibration isolation performance; namely, the acceleration values of accelerometers 6-1, 6-3, 6-4, and 6-6 are used as indicators to evaluate the vibration isolation performance. A smaller acceleration value indicates good vibration isolation performance. Conversely, a greater acceleration value leads to poor vibration isolation performance.

\section{Identification Model of Multisources Complex Excitations Active Vibration Isolation System}

Establishing the model of multisources complex excitations active vibration isolation system is the base and premise for the active vibration isolation. Modeling methods for the active vibration isolation system can be divided into two types: the first is analytical method, including multibody dynamics method, finite element method, and four terminal parameters' method, which make certain reasonable assumptions and provide description with accurate mathematical expression. For a more complex system, however, there are general cases where certain parameters cannot be determined and then meet the demand for modeling. The second is system identification method, which is suitable for the system whose mechanism is unclear or overly complex. For a system with unclear or overly complicated mechanism, the system model structure and parameters are determined by input/output data collected from the system under a certain condition, with no need to have a deep understanding of its internal mechanism, which is especially a complex one. The multisources complex excitations active vibration isolation system is a system that achieves the vibration isolation by the combined action of active and passive vibrationisolating elements, which is the typical mechatronics system. There is the strong nonlinearity due to the complex and changeable excitation signals and excitation sources intercouple via structures. Excitation sources and active vibration isolators intercouple with each other as well. Active and passive vibration isolators are also of features of coupling and nonlinearity. Besides, as the system is complex, it is hard to establish the mathematical model meeting requirements with the analytical method adopted. As the experiment data measured includes all the information related to the model, adopting the model identification method is an effective way to solve such issues. The least-squares system identification method is to determine the system model parameters with squares and functions of generalized error minimized by adopting the least-squares principle. The method can be used both for the linear and for the nonlinear systems, which is of features of concise principle, rapid convergence, being easy to implement, and so forth and is most widely used in the system identification. In addition, the recursive least-squares method considering both the amount of calculation and accuracy for the model identification of the multisources complex excitation active vibration isolation system is adopted. The experimental system of experimental data required by the identification model is shown in Figure 2, including the signal acquisition device MI-7016 (signal generator included), power amplifier of active vibration isolator, accelerometer, active vibration isolator, multisources excitation vibration isolation system, and PC. Firstly, use the signal generator of data collecting analyzer to produce the white-noise voltage signal as the input one for the power amplifier, which drives the active vibration isolator for action and causes vibration. Use the accelerometer to measure the vibration output of relevant position for the vibration isolation system. And adopt the data collecting analyzer to synchronously collect the input voltage and output acceleration signal with the sampling frequency of $2,048 \mathrm{~Hz}$. Then, identification models are obtained from experimental data using the frequencydomain identification method with the identification toolbox of system identification recursive least-squares method system in MATLAB. Based on demands of vibration isolation system, the frequency range of identification model is 10$200 \mathrm{~Hz}$.

The amplitude frequency curves and phase frequency curves of identification model and experimental data model for the input signal voltage of active vibration isolator 31 and output acceleration signal of accelerometer 6-2 are shown in Figure 3. The dash line indicates the curve of 


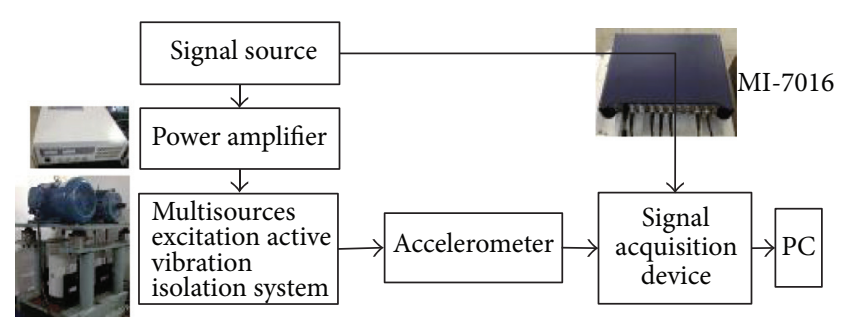

FIGURE 2: Data acquisition system of model identification experiment.

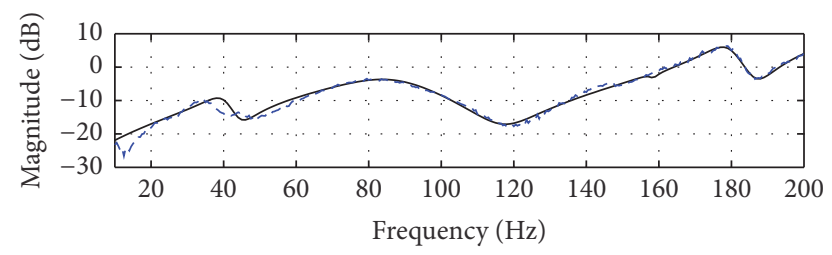

- Identification model

- - - Experimental model

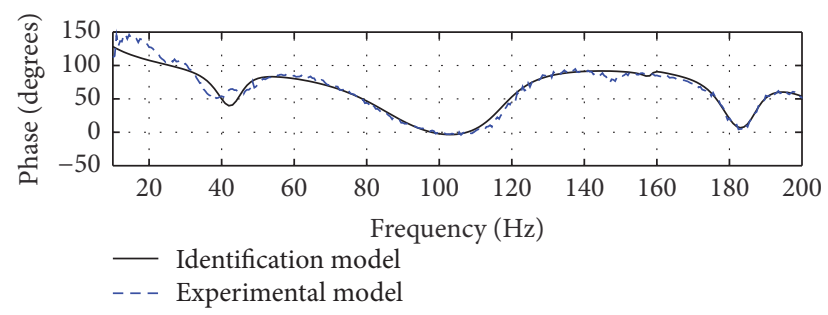

FIGURE 3: Frequency response curves of VA12 experiment model and identification system model.

experimental data model, while the solid line is for the curve of identification model, and the identification order is 10. The frequency response function of identification model marked as VA12 is shown in (1). And the degree of fitting for frequency response curves between the identification model and experimental model is $92.7 \%$. Hence,

$$
\mathrm{VA} 12=\frac{\text { num } 12}{\operatorname{den} 12},
$$

where

$$
\begin{aligned}
& \text { num12 }=\{0.311013499171883,-2.56012841901408, \\
& 9.55859708040467,-21.2396191368369, \\
& 30.9479185076993,-30.6625388620798, \\
& 20.6583763804234,-9.1280399491616, \\
& 2.40099885603445,-0.286579340218225\} ; \\
& \text { den12 }=\{1,-8.756169760397,35.3571162240213, \\
& -86.6357576983009,142.584991275251, \\
& -164.652521793695,135.099652939295, \\
& -77.7862010385311,30.0879691666081, \\
& -7.0641560134843,0.7651036085913\} .
\end{aligned}
$$
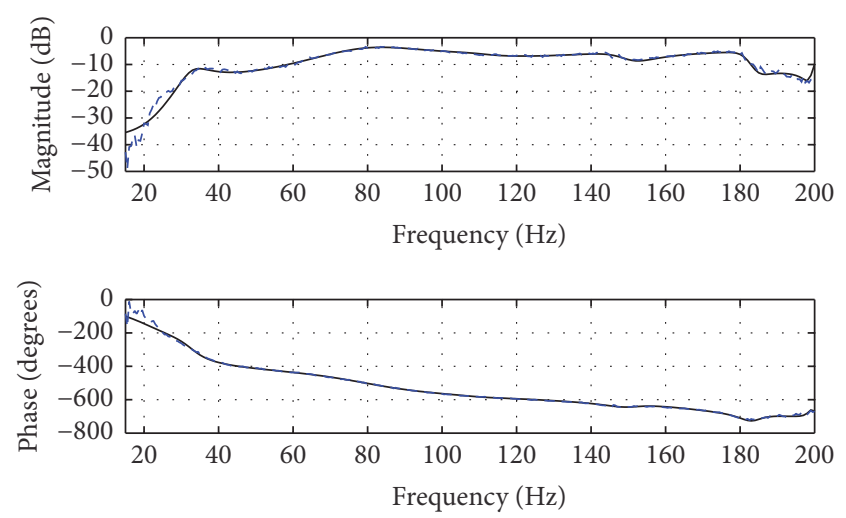

FIGURE 4: Frequency response curves of VA15 experiment model and identification system model.

The amplitude frequency curves and phase frequency curves of identification model and experimental data model for the input signal voltage of active vibration isolator 31 and output acceleration signal of accelerometer 6-5 are shown in Figure 4. The dash line indicates the curve of experimental data model, while the solid line is for the curve of identification model, and the identification order is 15. The frequency response function of identification model marked as VA15 is shown in (2). And the degree of fitting for frequency response curves between the identification model and experimental model is $93.6 \%$. Hence,

$$
\mathrm{VA} 15=\frac{\text { num } 15}{\operatorname{den} 15}
$$

where

$$
\begin{aligned}
& \text { num } 15=\{0.021099119109133, \\
& -0.266877217716250,1.563355590659295, \\
& -5.627965737654190,13.912054666944782, \\
& -24.963239501889934,33.476675755295993, \\
& -33.989410571624191,26.137574452525065, \\
& -15.038756179592204,6.293504077926695, \\
& -1.813947070617053,0.322675349706198, \\
& -0.026742728843002,0\} ; \\
& \text { den } 15=10 e 3 *\{0.001000000000000, \\
& -0.012297426536653,0.071348046461645, \\
& -0.258750388972723,0.655079645537215, \\
& -1.224442241448717,1.742214262508936, \\
& -1.916799666312638,1.638554585635531, \\
& -1.083050250259141,0.544928256480644, \\
& -0.202413305516768,0.052484051822397, \\
& -0.008505923096413,0.000650354060210\} .
\end{aligned}
$$

The amplitude frequency curves and phase frequency curves of identification model and experimental data model for the input signal voltage of active vibration isolator 32 and output acceleration signal of accelerometer 6-2 are 

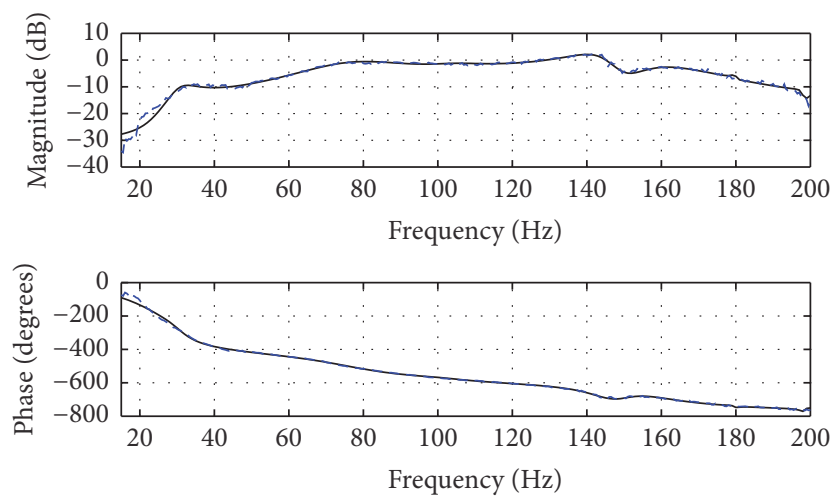

FIGURE 5: Frequency response curves of VA22 experiment model and identification system model.

shown in Figure 5. The dash line indicates the curve of experimental data model, while the solid line is for the curve of identification model, and the identification order is 11. The frequency response function of identification model marked as VA22 is shown in (3). And the degree of fitting for frequency response curves between the identification model and experimental model is $95.2 \%$. Hence,

$$
\mathrm{VA} 22=\frac{\operatorname{num} 22}{\operatorname{den} 22},
$$

where

$$
\begin{aligned}
& \text { num } 22=\{0.020255799459707, \\
& -0.250981092610675,1.440735766420271, \\
& -5.081416005504511,12.298196958765059, \\
& -21.582171098988812,28.263275634853709, \\
& -27.967143227985034,20.907065708048773, \\
& -11.656425965810755,4.707488560784323, \\
& -1.302452386121389,0.220854710747912, \\
& -0.017283355618937,0\} ; \\
& \text { den22 }=10 e 3 *\{0.001000000000000, \\
& -0.012464759154938,0.073237266230082, \\
& -0.268744276662871,0.687857790049814, \\
& -1.298769270976795,1.865205057373727, \\
& -2.069519289166297,1.782570893602977, \\
& -1.186141318858444,0.600226275160297, \\
& 0.224005376273297,0.058291837009602, \\
& -0.009469589515723,0.000724761388170\} .
\end{aligned}
$$

The amplitude frequency curves and phase frequency curves of identification model and experimental data model for the input signal voltage of active vibration isolator 32 and output acceleration signal of accelerometer 6-5 are shown in Figure 6. The dash line indicates the curve of experimental data model, while the solid line is for the curve of identification model, and the identification order is 15. The frequency response function of identification model
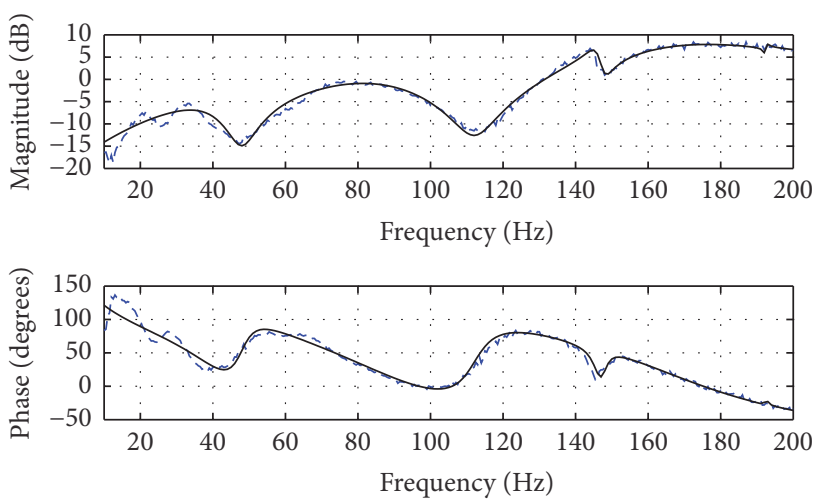

FIGURE 6: Frequency response curves of VA25 experiment model and identification system model.

marked as VA25 is shown in (4). And the degree of fitting for frequency response curves between the identification model and experimental model is $90.6 \%$. Hence,

$$
\text { VA25 }=\frac{\text { num } 25}{\operatorname{den} 25},
$$

where

$$
\begin{aligned}
& \text { num } 25=\{0,0.572058137397605, \\
& -4.740328881106740,17.8078898050623, \\
& -39.7966046974855,58.2954988049878, \\
& -58.0415116105133,39.2789734602179, \\
& -17.4240296360708,4.59828005401485, \\
& -0.550228206373591\} ; \\
& \text { den22 }=\{1,-8.7015002392363,34.8186972539061, \\
& -84.2994441133411,136.671489603445, \\
& -154.977752013698,124.450299426332, \\
& -69.8782666580524,26.2597938975302, \\
& -5.9658761201086,0.6225796563568\} .
\end{aligned}
$$

In addition, the models between the signal of accelerometer 6-2 and the signals of 6-1, 6-3, 6-4, and 6-6 accelerometers are, respectively, AA21, AA23, AA24, and AA26; the models between the 6-5 signal of accelerometer and the signals of 6-1, 6-3, 6-4, and 6-6 accelerometers are, respectively, AA51, AA53, AA54, and AA56. The approach to obtain identification model is consistent with the foregoing.

\section{PID Control Model and Improved PSO Algorithm}

4.1. The Establishment of PID Controller Model. Control is the core part of active vibration isolation system and can be divided into feedback control and feedforward control. Feedback control is applied to the situation in which there are many disturbance factors and they are not detectable. It is particularly suitable for the control of complex systems and systems with uncertain parameters. In terms of multisources 


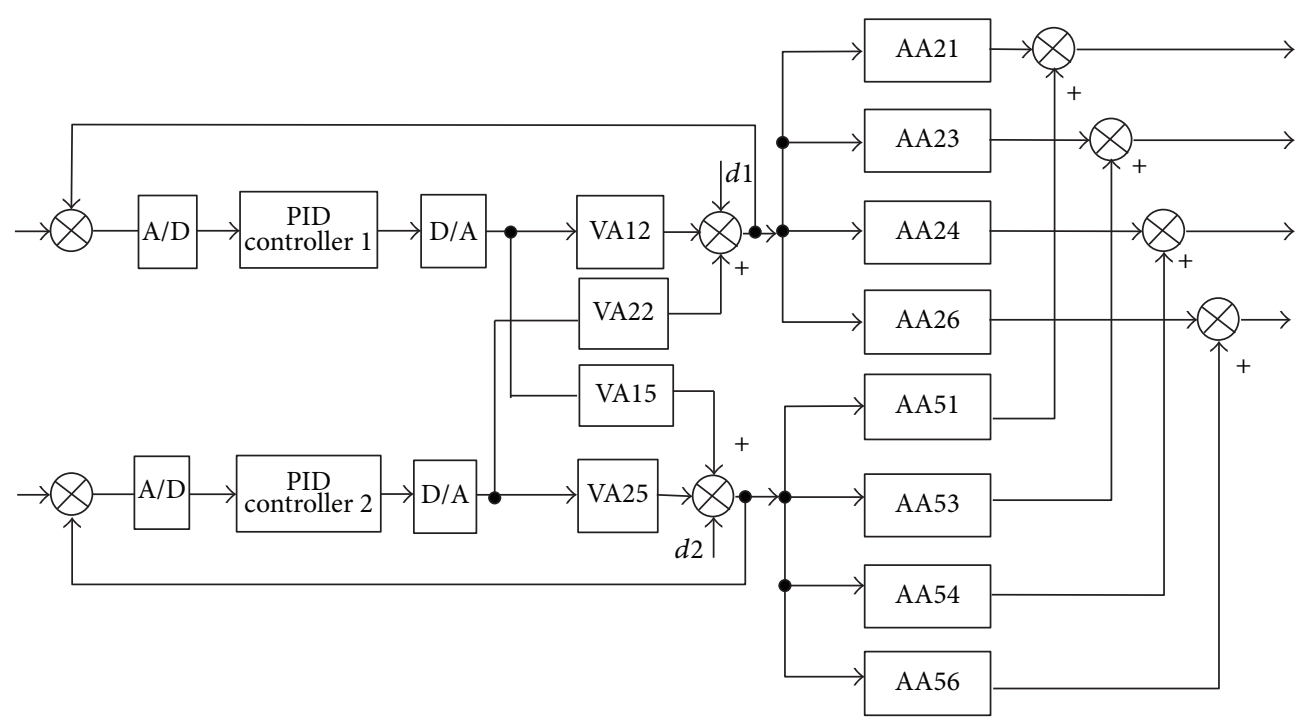

FIGURE 7: Control schematic diagram of multisources complex excitations active vibration isolation system.

excitation active vibration isolation system, the system is complicated and has complex disturbance signals. Therefore, feedback control is suitable for the system. Furthermore, PID controller is the most widely used feedback controller. It has the features of simple structure, good stability, reliable working performance, and strong robustness. Particularly, when the structure and parameters of the controlled object cannot be fully grasped or when precise mathematical model cannot be obtained, feedback controller also has very good control effect. The control effect of PID controller mainly depends on the three parameters of P, I, and D. Determining the control parameters is the main content of designing PID control system. Conventional PID controller obtains the PID control parameters by means of manual adjustment which is time-consuming and does not guarantee the best performance. The PID controller based on intelligent and improved algorithm obtains PID control parameters through the improvement of intelligent algorithm by means of integrating the PID controller with modern intelligent algorithms such as genetic algorithm, ant colony algorithm, and PSO. Compared with conventional PID control, it has the abilities of self-learning and self-adaption. Such PID controller can automatically identify the parameters of controlled process and adapt to the changes of the parameters of controlled process. Besides, like the conventional PID controller, it also has the features of simple structure, good stability, reliable working performance, and strong robustness. Compared with other intelligent algorithms, PSO has the advantages of simple structure, fewer parameters, fast convergence, easy implementation, and so forth. For this reason, the PID controller of multisources complex excitations active vibration isolation system based on PSO is established. The purpose of active vibration isolation is to reduce the transmission of vibration acceleration, so the acceleration signals of 6-2 and 6-5 are as the feedback signals of PID controller. The controller carries out calculation and feedback output based on the control parameters obtained through the improved PSO algorithm. The amplified output signal regulates the current in active vibration isolation electromagnetic coil to generate a controllable electromagnetic force, and then active control can be conducted for multisources vibration. The control schematic diagram is shown in Figure 7.

4.2. Improved PSO Algorithm. PSO algorithm has the advantages of simple structure, fewer parameters, fast convergence, easy implementation, and so forth. Its space complexity and time complexity are relatively low, and it has been proved that good optimal solutions can be obtained with relatively small calculation cost [18-21]. It has been widely studied and applied in the field of optimizing PID parameters [22-24].

In PSO algorithm, each particle represents a potential solution to the problem, and each particle corresponds to a fitness value determined by the fitness function. The speed of the particles determines the direction and distance of the movement of those particles. In $D$-dimensional searching space, $\mathbf{X}=\left(\mathbf{X}_{1}, \mathbf{X}_{2}, \ldots, \mathbf{X}_{i}, \ldots, \mathbf{X}_{N}\right)$ represents the population that consists of $N$ particles; $\mathbf{X}_{i}=\left(X_{i 1}, X_{i 2}, \ldots, X_{i D}\right)^{T}$ represents the position of number $i$ particle in the searching space, that is, a potential solution; $\mathbf{V}_{i}=\left(V_{i 1}, V_{i 2}, \ldots, V_{i D}\right)^{T}$ represents the speed of number $i$ particle; $\mathbf{P}_{i}=\left(P_{i 1}, P_{i 2}, \ldots, P_{i D}\right)^{T}$ is the best optimum searched of number $i$ particle so far; $\mathbf{P}_{g}=\left(P_{g 1}, P_{g 2}, \ldots, P_{g D}\right)^{T}$ is the optimum position searched by the entire particle swarm so far. In each iteration project, particles update their speeds and positions through individual extremum and group extremum; that is,

$$
\begin{aligned}
& X_{i d}^{k+1}=X_{i d}^{k+1}+V_{i d}^{K+1} \\
& V_{i d}^{k+1}=\omega V_{i d}^{k}+c_{1} r_{1}\left(P_{i d}^{k}-X_{i d}^{k}\right)+c_{2} r_{2}\left(P_{g d}^{k}-X_{g d}^{k}\right),
\end{aligned}
$$

where $\omega$ is inertia weight factor; $k$ is the current number of evolution generations; $i=1,2, \ldots, N$ is the number of particles; $d=1,2, \ldots, D$ is each particle dimension; $c_{1}$ and $c_{2}$ are the acceleration constants; $r_{1}$ and $r_{2}$ are random numbers in $[0,1]$. 


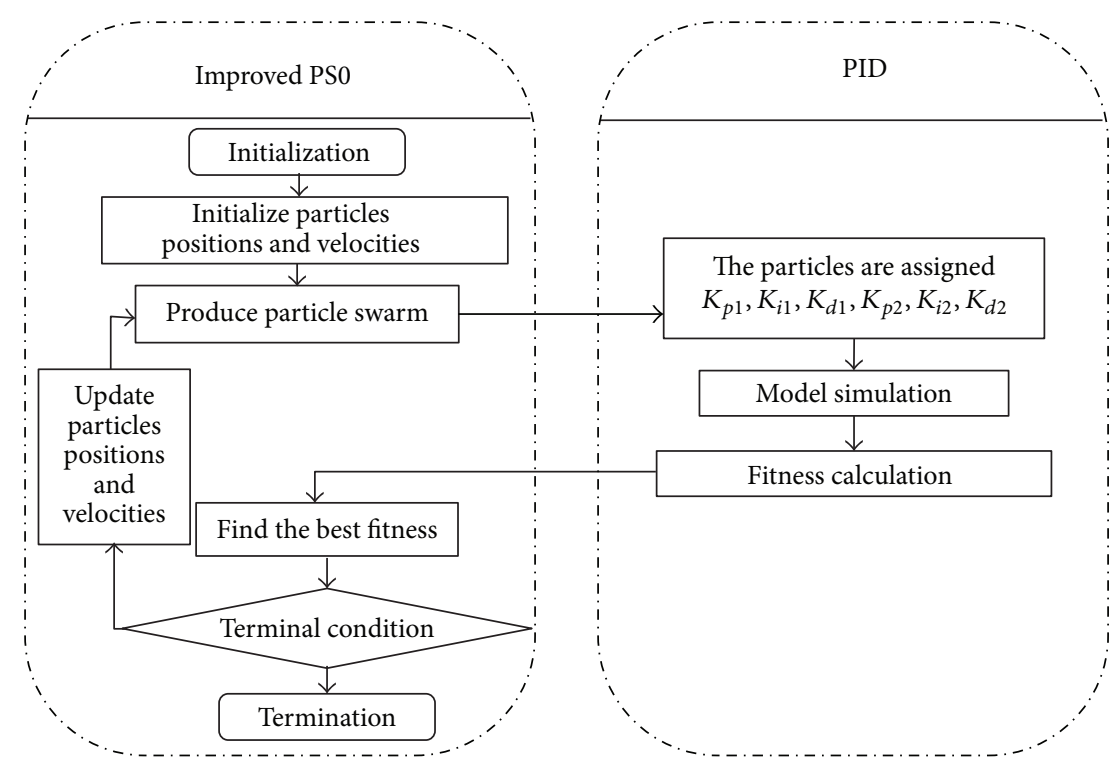

FIGURE 8: Optimization process for PID using improved PSO.

Inertia weight factor $\omega$ reflects the particle's ability to inherit the previous speed. Greater weight is in favor of the global search, and smaller weight is to the benefit of local search. The inertia factors of conventional PSO algorithm are constants and cannot give consideration to the abilities of both global search and local search. In order to achieve better global searching and local searching capabilities of balancing algorithm, this article puts forward an improved PSO algorithm of dynamic inertia weight factor. Inertia weight factor $\omega$ is defined as a function relevant to the evolution generation $k$. The function is

$$
\omega(k)=\omega_{\text {start }}-\left(\omega_{\text {start }}-\omega_{\text {end }}\right)\left(\frac{k}{T_{\max }}\right)^{2},
$$

where $\omega_{\text {start }}$ is the greater value of initial inertia weight, which maintains relatively strong global searching ability for the algorithm; $\omega_{\text {end }}$ is the smaller value of end inertia weight, which ensures relatively strong local searching ability. In addition, in the improved method of weighting factors proposed by (7), weight factor $\omega$ changes slowly in the early stage and maintains a larger value, ensuring the global searching ability of the algorithm; in later stage, the weight factor $\omega$ changes fast and significantly increases the local optimizing ability of the algorithm, which guarantees a good solution performance.

\subsection{PID Control Parameters Optimization Based on Improved} PSO Algorithm. The optimization of PID controller is to determine a suitable set of parameters $K_{p}, K_{i}$, and $K_{d}$ that can make the vibration isolation performance indicator optimal.

The bridge between improved PSO algorithm and PID control model is the particle and the corresponding fitness value of the particle. The particle is the parameters of PID controllers, and the format of particle is $\left[K_{p 1}, K_{i 1}, K_{d 1}, K_{p 2}, K_{i 2}, K_{d 2}\right]$. The corresponding fitness value of the particle is the performance indicator of control system.
Based on the evaluation criteria of isolation effect, the sum of ISE (integral square errors) of four acceleration values is as the fitness function, and it is written as

$$
F=\int_{0}^{+\infty}\left(\left|a_{1}(t)^{2}\right|+\left|a_{2}(t)^{2}\right|+\left|a_{3}(t)^{2}\right|+\left|a_{4}(t)^{2}\right|\right) d t
$$

The optimization process of PID parameters based on improved PSO is as follows.

Step 1. Set up the optimum fitness and maximum number of evolution generations to determine the termination condition: the optimum fitness is achieved, or the maximum evolution generation number is reached.

Step 2. Initial particle swarm is generated randomly. Determine the number of initial populations, the maximum speed and minimum speed of particle swarm, and the positions and speeds of all randomly generated particles. Set up the upper and lower limits of particle parameters to determine the initial inertia weights at the beginning and at the end of the improved PSO algorithm.

Step 3. Assign the value of the particles to PID control parameters $\left[K_{p 1}, K_{i 1}, K_{d 1}, K_{p 2}, K_{i 2}, K_{d 2}\right]$ in turn, and then operate PID control model to obtain the corresponding fitness values of that group.

Step 4. Transmit the fitness values to PSO as the adaptation value of the particle. Find the best fitness value to see whether the termination condition is satisfied. If so, exit the program.

Step 5. If the termination condition is not satisfied, use the algorithm of (5) to update the positions and speeds of particles. New particle swarm will be generated, and the number of evolution generations will increase by 1 . Return to Step 3.

Detailed optimization process of PID parameters based on improved PSO can be found in Figure 8. 


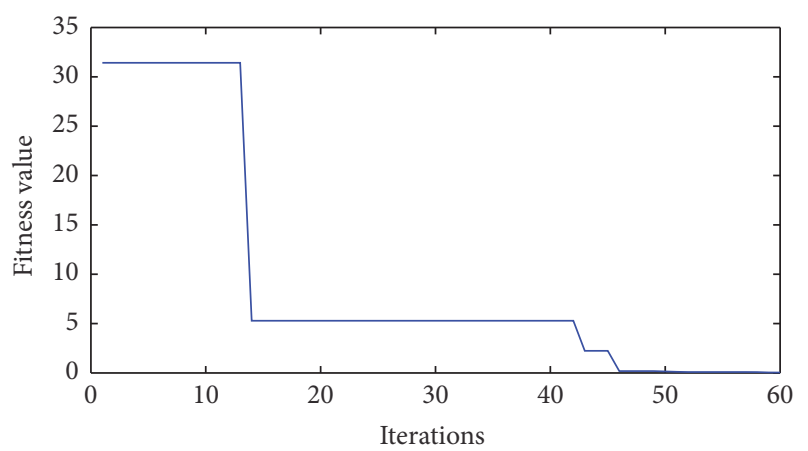

FIGURE 9: Improved PSO iterative process and the optimum fitness value.

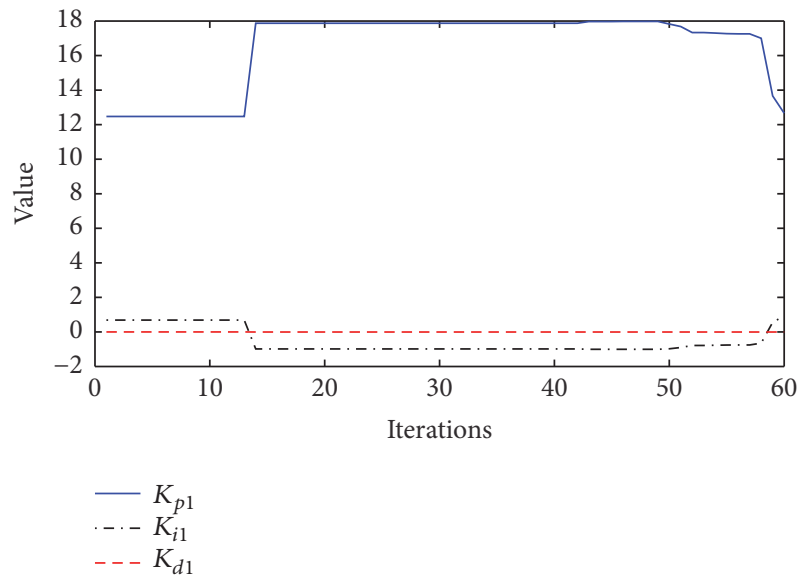

FIGURE 10: Improved PSO iterative process and the parameter values of PID controller 1 .

\section{Simulation}

5.1. Optimization of Parameters. Optimization for the parameters of PID controllers based on improved PSO is carried out. Initial particle swarms are generated randomly, and the number of initial particle swarms is 100 . In terms of the positions and speeds of all particles generated randomly, the largest and smallest speeds of particle swarms are 1 and -1 , and the best fitness value setting is $10 e-4$. Based on experience, the upper limit of setting up the parameters is $[20,1.8000,0.0100,5$, $0.5,0.05]$, and the lower limit is $[6,-1.8000,-0.0114,0.1,-1$, $-0.05]$. The maximum number of iterations (evolution generations) is 60 . The inertia weights at the beginning and at the end are 0.9 and 0.4. After 60 iterations, the optimum fitness value is $4.38 e-2$ as shown in Figure 9. The values of optimal individual are [12.664217059535254 0.924957088184704 $-0.0044146773341793 .598106664977562 \quad-0.953201094794553$ -0.001237398124662 ] as shown in Figures 10 and 11.

5.2. Control Simulation Results. There are multiple excitation sources and complex interference signals of active isolation system. In order to determine whether the established PID controllers and control parameters obtained through optimization are valid, simulation analysis for active isolation system is carried out under the double sine interference signals and double linear swept sine interference signals based on actual conditions.

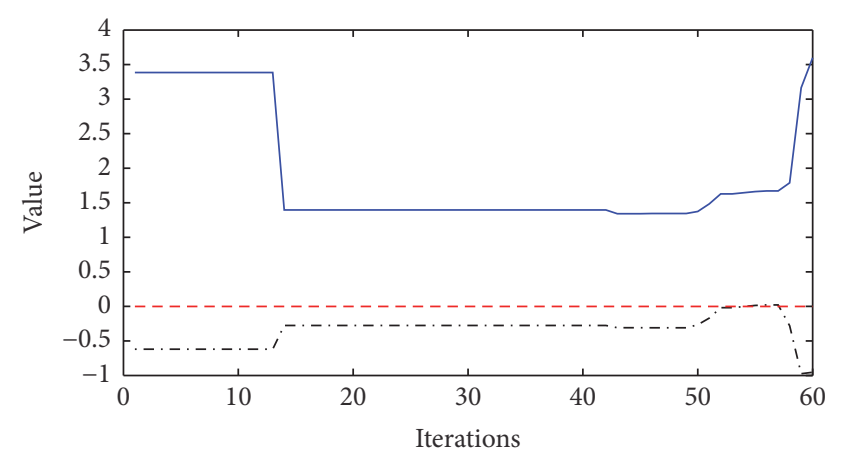

$$
\begin{aligned}
& -K_{p 2} \\
& -\cdot-K_{i 2} \\
& ---K_{d 2}
\end{aligned}
$$

FIGURE 11: Improved PSO iterative process and the parameter values of PID controller 2.
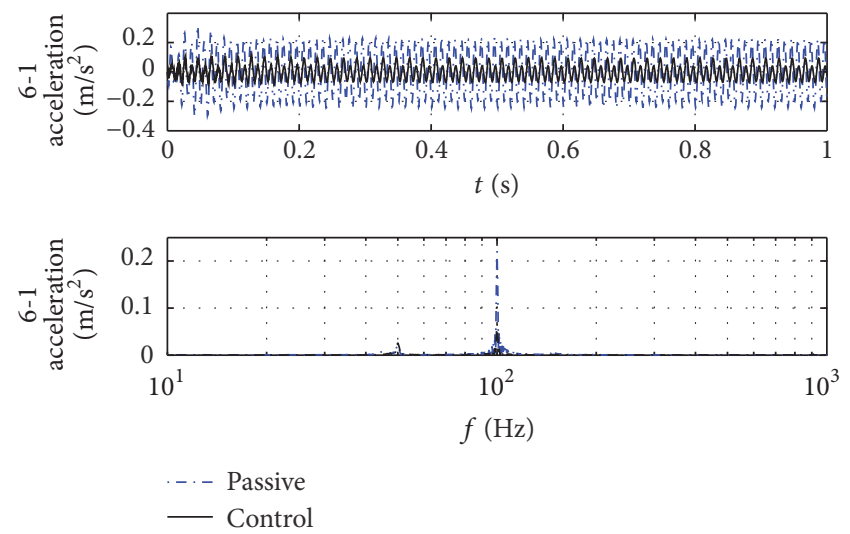

FIGURE 12: Comparison time-frequency domain curves of acceleration response of position 6-1 on the isolation system before and after being controlled under double sinusoidal interference.

5.2.1. Double Sine Interference Signals. Taking the actual operating conditions into account, when both motor vibration sources are sinusoidal signals, the 1-1 source signal is $50 \mathrm{~Hz}$ sinusoidal signal, and the $1-2$ source signal is $100 \mathrm{~Hz}$ sinusoidal signal. By using the control parameters obtained through the optimization of improved PSO algorithm, the isolation effects before and after control of the isolation system are analyzed, and the simulation time is $1 \mathrm{~s}$. Figures 12-15 are the comparison curves of acceleration responses of the 6-1 to 6-4 positions in multiexcitation vibration isolation system before and after being controlled under the interference of double sine signals.

The time-frequency domain curves of the output acceleration of the 6-1 position in the isolation system before and after being controlled are shown in Figure 12. In the time domain curves, the vertical axis represents the magnitude of the acceleration response, and the horizontal axis is time. The dotted line represents the response curve of acceleration before being controlled (passive), and the solid line is the response curve of acceleration after being controlled (active). It can be seen that the maximum peak-to-peak amplitude of acceleration response is reduced from passive system's 

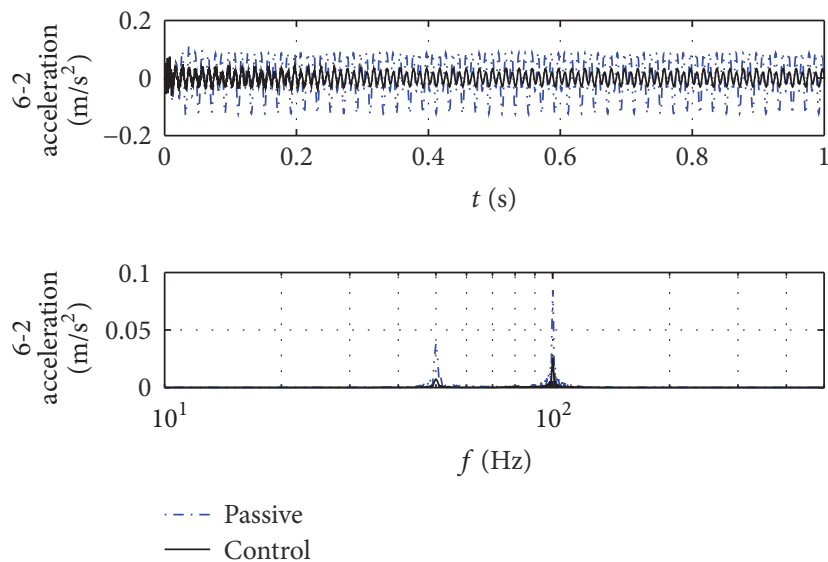

FIGURE 13: Comparison time-frequency domain curves of acceleration response of position 6-2 on the isolation system before and after being controlled under double sinusoidal interference.
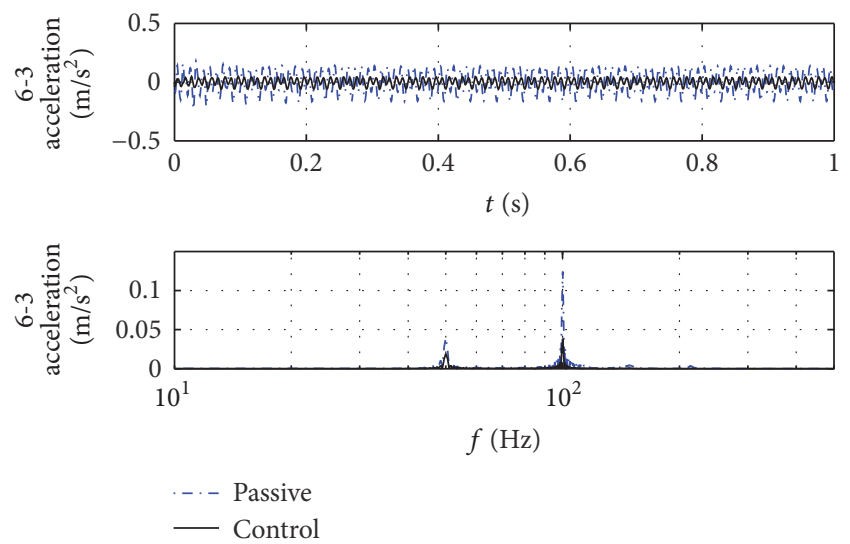

FIGURE 14: Comparison time-frequency domain curves of acceleration response of position 6-3 on the isolation system before and after being controlled under double sinusoidal interference.

$0.62 \mathrm{~m} / \mathrm{s}^{2}$ without the control to active system's $0.17 \mathrm{~m} / \mathrm{s}^{2}$ under the control. The peak of active system's maximum acceleration response is only $1 / 4$ of passive system. In the frequency domain curves, the vertical axis represents the magnitude of the acceleration response, and the horizontal axis is frequency. Likewise, the dotted line represents the response curve of acceleration before being controlled (passive), and the solid line is the response curve of acceleration after being controlled (active). It can be discovered that, at $100 \mathrm{~Hz}$, acceleration amplitude is reduced from $0.22 \mathrm{~m} / \mathrm{s}^{2}$ without the control to $0.066 \mathrm{~m} / \mathrm{s}^{2}$ under the control; the amplitude is reduced by $2 / 3$. After being controlled at $50 \mathrm{~Hz}$, there is also corresponding decrease in terms of the amplitude.

The time-frequency domain curves of the output acceleration of the 6-2 position in the isolation system before and after being controlled are shown in Figure 13. In the time domain curves, the vertical axis represents the magnitude of the acceleration response, and the horizontal axis is time. The dotted line represents the response curve of acceleration before being controlled (passive), and the solid line is the response curve of acceleration after being controlled (active). It can be seen that the maximum peak-to-peak amplitude
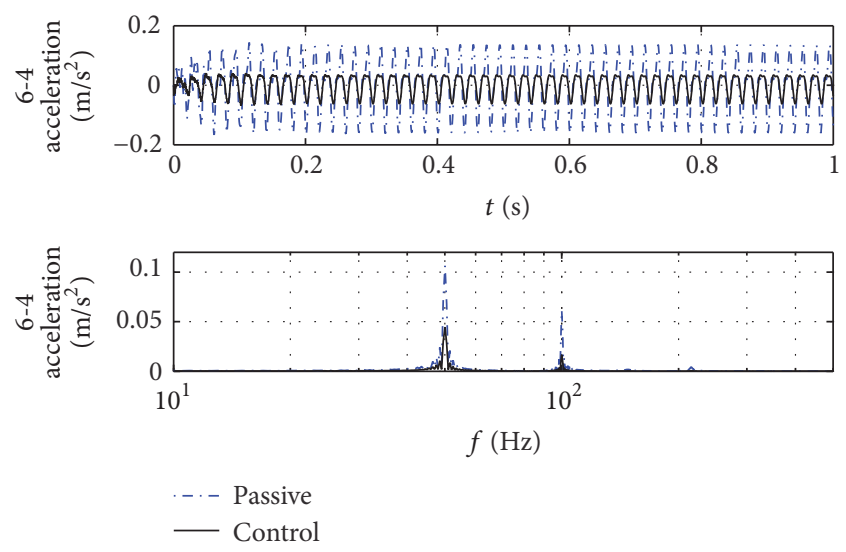

FIGURE 15: Comparison time-frequency domain curves of acceleration response of position 6-4 on the isolation system before and after being controlled under double sinusoidal interference.

of acceleration response is reduced from passive system's $0.24 \mathrm{~m} / \mathrm{s}^{2}$ without the control to active system's $0.073 \mathrm{~m} / \mathrm{s}^{2}$ under the control. The peak of active system's maximum acceleration response is less than $1 / 3$ of passive system. In the frequency domain curves, the vertical axis represents the magnitude of the acceleration response, and the horizontal axis is frequency. Likewise, the dotted line represents the response curve of acceleration before being controlled (passive), and the solid line is the response curve of acceleration after being controlled (active). It can be discovered that acceleration amplitude is reduced from $0.085 \mathrm{~m} / \mathrm{s}^{2}$ before being controlled to $0.026 \mathrm{~m} / \mathrm{s}^{2}$ after being controlled; the amplitude is reduced by around $70 \%$. At $50 \mathrm{~Hz}$, the acceleration response amplitude reduces from $0.038 \mathrm{~m} / \mathrm{s}^{2}$ without the control to $0.0076 \mathrm{~m} / \mathrm{s}^{2}$ under the control; the amplitude is reduced by around $80 \%$.

The time-frequency domain curves of the output acceleration of the 6-3 position in the isolation system before and after being controlled are shown in Figure 14. In the time domain curves, the vertical axis represents the magnitude of the acceleration response, and the horizontal axis is time. The dotted line represents the response curve of acceleration before being controlled (passive), and the solid line is the response curve of acceleration after being controlled (active). It can be seen that the maximum peak-to-peak amplitude of acceleration response is reduced from passive system's $0.41 \mathrm{~m} / \mathrm{s}^{2}$ without the control to active system's $0.107 \mathrm{~m} / \mathrm{s}^{2}$ under the control. The peak of active system's maximum acceleration response is only about $1 / 4$ of passive system. In the frequency domain curves, the vertical axis represents the magnitude of the acceleration response, and the horizontal axis is frequency. Likewise, the dotted line represents the response curve of acceleration before being controlled (passive), and the solid line is the response curve of acceleration after being controlled (active). It can be discovered that, at $100 \mathrm{~Hz}$, acceleration amplitude is reduced from $0.124 \mathrm{~m} / \mathrm{s}^{2}$ before being controlled to $0.038 \mathrm{~m} / \mathrm{s}^{2}$ after being controlled; the amplitude is reduced by around $60 \%$. At $50 \mathrm{~Hz}$, the acceleration response amplitude reduces from $0.040 \mathrm{~m} / \mathrm{s}^{2}$ 
without the control to $0.019 \mathrm{~m} / \mathrm{s}^{2}$ under the control; the amplitude is reduced by more than $50 \%$.

The time-frequency domain curves of the output acceleration of the 6-4 position in the isolation system before and after being controlled are shown in Figure 15. In the time domain curves, the vertical axis represents the magnitude of the acceleration response, and the horizontal axis is time. The dotted line represents the response curve of acceleration before being controlled (passive), and the solid line is the response curve of acceleration after being controlled (active). It can be seen that the maximum peak-to-peak amplitude of acceleration response is reduced from passive system's $0.31 \mathrm{~m} / \mathrm{s}^{2}$ without the control to active system's $0.097 \mathrm{~m} / \mathrm{s}^{2}$ under the control. The peak of active system's maximum acceleration response is less than $1 / 3$ of passive system. In the frequency domain curves, the vertical axis represents the magnitude of the acceleration response, and the horizontal axis is frequency. Likewise, the dotted line represents the response curve of acceleration before being controlled (passive), and the solid line is the response curve of acceleration after being controlled (active). It can be discovered that, at $50 \mathrm{~Hz}$, acceleration amplitude is reduced from $0.106 \mathrm{~m} / \mathrm{s}^{2}$ before being controlled to $0.0446 \mathrm{~m} / \mathrm{s}^{2}$ after being controlled; the amplitude is reduced by around $60 \%$. At $100 \mathrm{~Hz}$, the acceleration response amplitude reduces from $0.061 \mathrm{~m} / \mathrm{s}^{2}$ without the control to $0.016 \mathrm{~m} / \mathrm{s}^{2}$ under the control; the amplitude is reduced by more than $75 \%$.

5.2.2. Double Linear Swept Sine Interference Signals. Given the complexity of the actual interference, in order to further validate the effectiveness of the PID control model of multiple excitation active vibration isolation system and PID control parameters obtained through the optimization of improved PSO algorithm, and considering the actual operating conditions, simulation analysis is carried out against the linear swept sine signals when the 1-1 and 1-2 vibration source signals are both $10-200 \mathrm{~Hz}$ under the situation that both motor vibration sources are linear swept sine signals. The simulation time is $1 \mathrm{~s}$. Figures 16-19 are the comparison curves of acceleration responses of the 6-1 to 6-4 positions in multiexcitation vibration isolation system before and after being controlled under the interference of double linear swept sine signals.

The time-frequency domain curves of the output acceleration of position 6-1 in the isolation system before and after being controlled under the double linear swept sine interference signals are shown in Figure 16. In the time domain curves, the vertical axis represents the magnitude of the acceleration response, and the horizontal axis is time. The dotted line represents the response curve of acceleration before being controlled, and the solid line is the response curve of acceleration after being controlled. It can be seen that the maximum peak-to-peak amplitude of acceleration response is reduced from passive system's $3.52 \mathrm{~m} / \mathrm{s}^{2}$ without the control to active system's $0.34 \mathrm{~m} / \mathrm{s}^{2}$ under the control. The peak of active system's maximum acceleration response is only $1 / 10$ of passive system. In the frequency domain curves, the vertical axis represents the magnitude of the acceleration response, and the horizontal axis is time. Likewise, the dotted
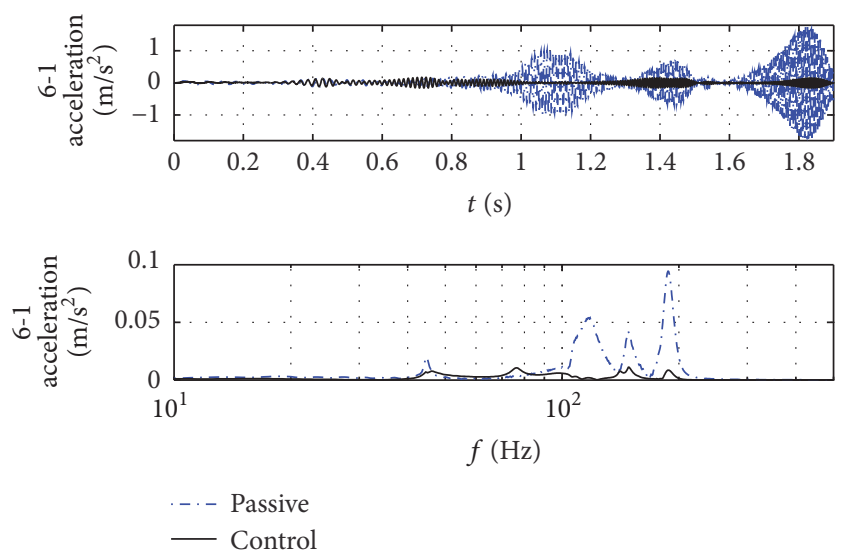

FIGURE 16: Comparison time-frequency domain curves of acceleration response of position 6-1 on the isolation system before and after being controlled under double linear swept sine interference.
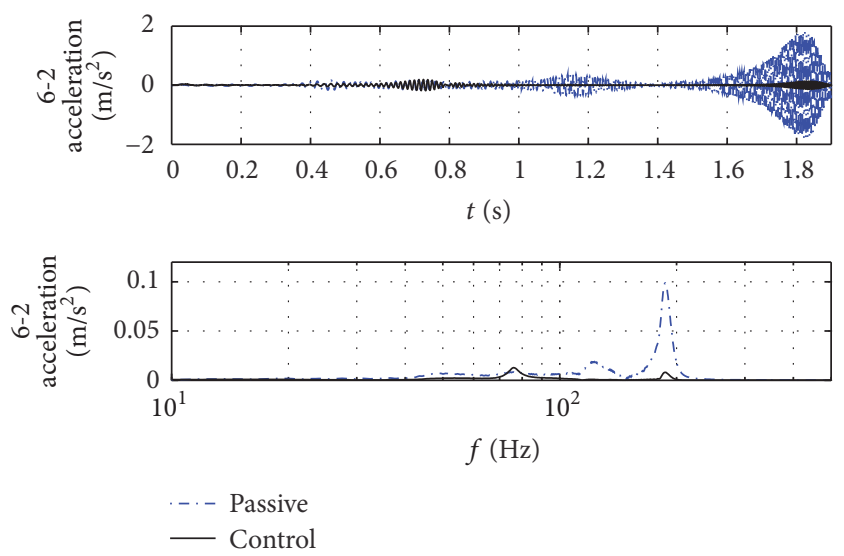

FIGURE 17: Comparison time-frequency domain curves of acceleration response of position 6-2 on the isolation system before and after being controlled under double linear swept sine interference.

line represents the response curve of acceleration before being controlled, and the solid line is the response curve of acceleration after being controlled. It can be discovered that the peak of maximum acceleration response is reduced from $0.094 \mathrm{~m} / \mathrm{s}^{2}$ before being controlled to $0.011 \mathrm{~m} / \mathrm{s}^{2}$ after being controlled. It is reduced by about $90 \%$. The peaks of other frequency ranges are also significantly reduced, and the increase of isolation effect is obvious.

The time-frequency domain curves of the output acceleration of position 6-2 in the isolation system before and after being controlled under the double linear swept sine interference signals are shown in Figure 17. In the time domain curves, the dotted line represents the response curve of acceleration before being controlled, and the solid line is the response curve of acceleration after being controlled. It can be seen that the maximum peak-to-peak amplitude of acceleration response is reduced from passive system's $3.61 \mathrm{~m} / \mathrm{s}^{2}$ without the control to active system's $0.378 \mathrm{~m} / \mathrm{s}^{2}$ under the control. The peak of active system's maximum acceleration response is only around $1 / 10$ of passive system. In the frequency domain curves, the dotted line represents the response curve of acceleration before being controlled, and the solid line is 

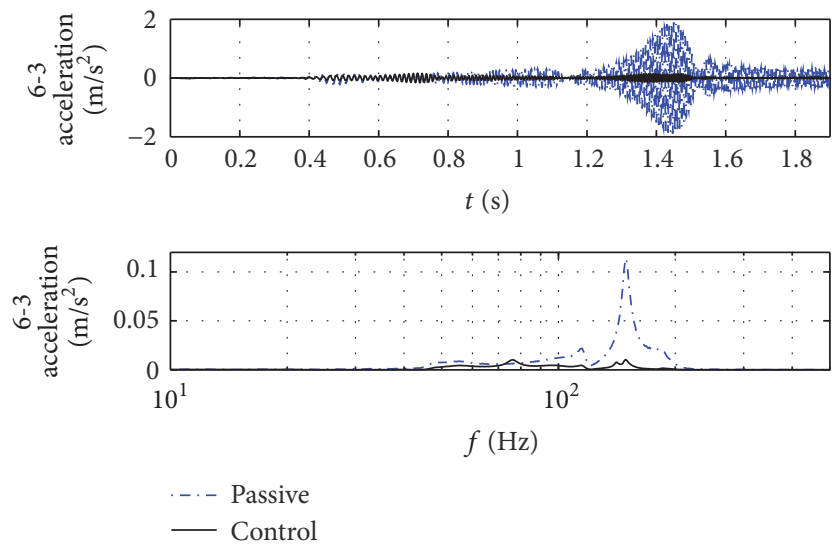

FIgURE 18: Comparison time-frequency domain curves of acceleration response of position 6-3 on the isolation system before and after being controlled under double linear swept sine interference.
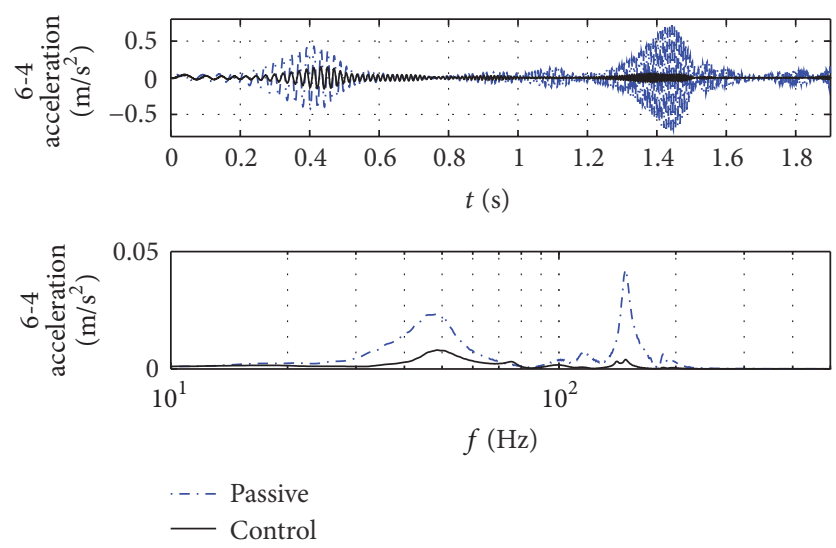

FIGURE 19: Comparison time-frequency domain curves of acceleration response of position 6-4 on the isolation system before and after being controlled under double linear swept sine interference.

the response curve of acceleration after being controlled. It can be discovered that the peak of maximum acceleration response is reduced from $0.114 \mathrm{~m} / \mathrm{s}^{2}$ before being controlled to $0.0102 \mathrm{~m} / \mathrm{s}^{2}$ after being controlled. It is reduced by more than $90 \%$. The peaks of other frequency ranges are also significantly reduced, and the increase of isolation effect is obvious.

The time-frequency domain curves of the output acceleration of position 6-3 in the isolation system before and after being controlled under the double linear swept sine interference signals are shown in Figure 18. In the time domain curves, the dotted line represents the response curve of acceleration before being controlled, and the solid line is the response curve of acceleration after being controlled. It can be seen that the maximum peak-to-peak amplitude of acceleration response is reduced from passive system's $3.77 \mathrm{~m} / \mathrm{s}^{2}$ without the control to active system's $0.324 \mathrm{~m} / \mathrm{s}^{2}$ under the control. The peak of active system's maximum acceleration response is less than $1 / 10$ of passive system. In the frequency domain curves, the dotted line represents the response curve of acceleration before being controlled, and the solid line is the response curve of acceleration after being

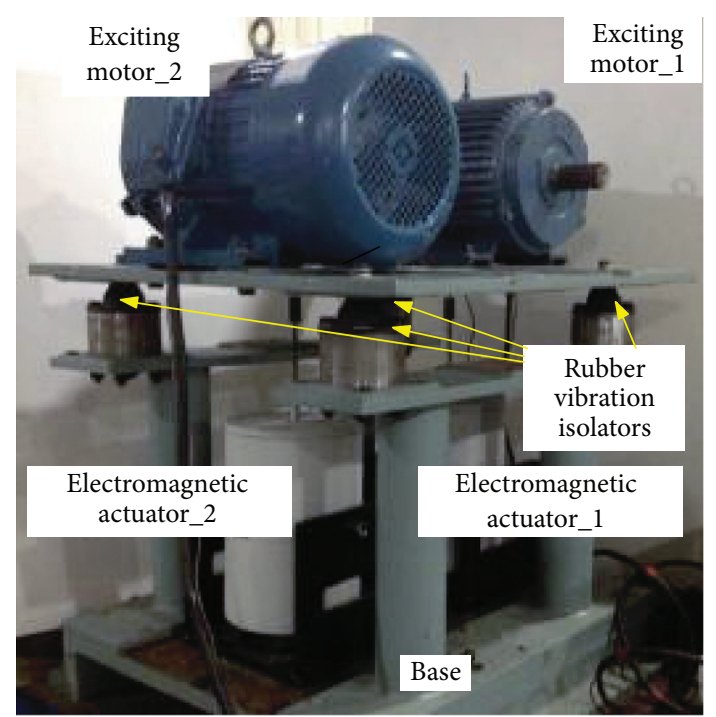

FIGURE 20: Multisources complex excitations active vibration isolation experimental platform.

controlled. It can be discovered that the peak of maximum acceleration response is reduced from $0.0987 \mathrm{~m} / \mathrm{s}^{2}$ before being controlled to $0.0126 \mathrm{~m} / \mathrm{s}^{2}$ after being controlled. It is reduced by around $90 \%$. The peaks of other frequency ranges are also significantly reduced, and the increase of isolation effect is obvious.

The time-frequency domain curves of the output acceleration of position 6-4 in the isolation system before and after being controlled under the double linear swept sine interference signals are shown in Figure 19. In the time domain curves, the dotted line represents the response curve of acceleration before being controlled, and the solid line is the response curve of acceleration after being controlled. It can be seen that the maximum peak-to-peak amplitude of acceleration response is reduced from passive system's $1.44 \mathrm{~m} / \mathrm{s}^{2}$ without the control to active system's $0.302 \mathrm{~m} / \mathrm{s}^{2}$ under the control. The peak of active system's maximum acceleration response is around $1 / 5$ of passive system. In the frequency domain curves, the dotted line represents the response curve of acceleration before being controlled, and the solid line is the response curve of acceleration after being controlled. It can be discovered that the peak of maximum acceleration response is reduced from $0.0423 \mathrm{~m} / \mathrm{s}^{2}$ before being controlled to $0.00803 \mathrm{~m} / \mathrm{s}^{2}$ after being controlled. It is reduced by more than $80 \%$. The peaks of other frequency ranges are also significantly reduced, and the increase of isolation effect is obvious.

\section{Experimental Verification}

6.1. The Establishment of Experimental Platform. In order to further validate the effectiveness of the PID control model of multisources complex excitations active vibration isolation system and the PID control parameters obtained through the improved PSO algorithm, an experimental platform of multiple excitation active vibration isolation system is established, as shown in Figure 20. The experimental platform includes 


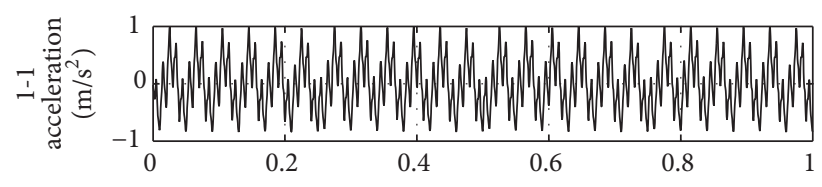

$t(\mathrm{~s})$

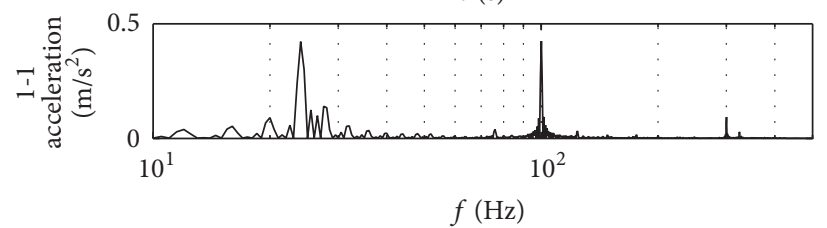

(a) Time-frequency domain curves of the acceleration signal in 6-2 motor position

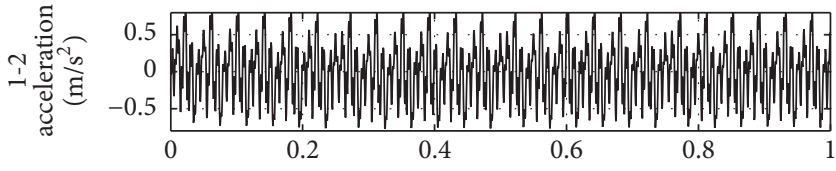

$t(\mathrm{~s})$

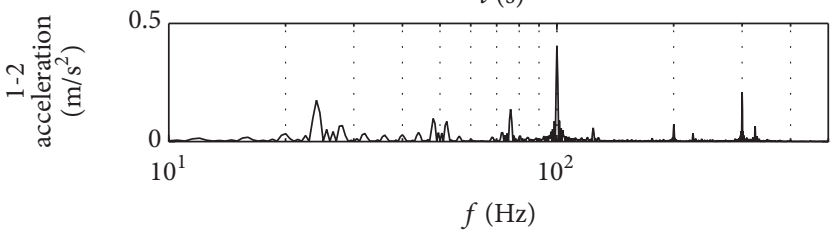

(b) Time-frequency domain curves of the acceleration signal in 6-5 motor position

FIGURE 21: Actual vibration acceleration signals of two excitation motors.

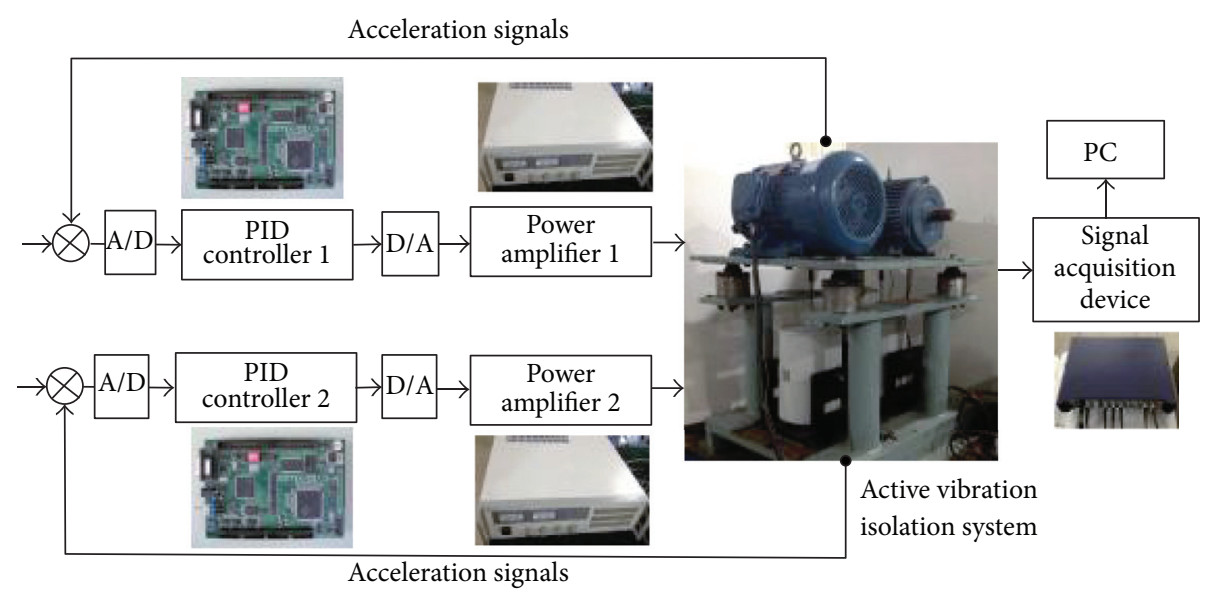

FIGURE 22: Control block diagram of multiexcitation active vibration isolation experiment platform.

two vibration motors and transducers, two electromagnetic actuators and amplifiers, four rubber isolators, bases, DSP controllers, and accelerometers. The experimental platform is supported by the parallel connection of four rubber isolators and two electromagnetic active isolators. The experiment platform uses two excitation motors to conduct excitation, and the rotation speeds of two motors are $1500 \mathrm{r} / \mathrm{min}$ and $3000 \mathrm{r} / \mathrm{min}$, respectively. The actual acceleration signals of the two motors are measured by accelerometer 6-2 and accelerometer 6-5, which are shown in Figure 21, wherein Figure 21(a) is the time-frequency curve of the acceleration of position 6-2; Figure 21(b) is the time-frequency curve of the acceleration of position 6-5. It can be seen that, due to the existence of motor frequency coupling, the vibration frequencies of the two positions mainly concentrate in the vicinity of $25 \mathrm{~Hz}, 50 \mathrm{~Hz}, 100 \mathrm{~Hz}$, and $200 \mathrm{~Hz}$. In the active vibration control procedure of isolation platform, the inputs of PID controllers are the acceleration signals of the corresponding position on the isolation platform. Based on the control parameters obtained through the improved PSO algorithm, the controllers carried out calculation and output control signals. The control signals go through the power amplifiers to regulate the electric currents in the active vibration isolation electromagnetic coil to generate a controllable electromagnetic force. In this case, it carries out vibration active control of multisources vibration. The acceleration of each position is acquired by using signal acquisition instrument, and the specific control model is shown in Figure 22.

6.2. Comparison of Experimental Results. By using the established PID control model and the improved PSO algorithm, the parameters of control system are obtained. The acceleration response amplitudes of positions 6-1 to 6-4 on the experiment platform before and after being controlled are shown in Figures 23-26.

The time-frequency domain curves of the output acceleration of position 6-1 in the isolation experimental platform before and after being controlled are shown in Figure 23. In the time domain curves, the vertical axis represents the magnitude of the acceleration response, and the horizontal axis is time. The dotted line represents the response curve of acceleration before being controlled, and the solid line is the response curve of acceleration after being controlled. It can be seen that the maximum peak-to-peak amplitude of acceleration response is reduced from passive system's $0.5415 \mathrm{~m} / \mathrm{s}^{2}$ before being controlled to active system's $0.1839 \mathrm{~m} / \mathrm{s}^{2}$ after being controlled. The maximum acceleration response of the 

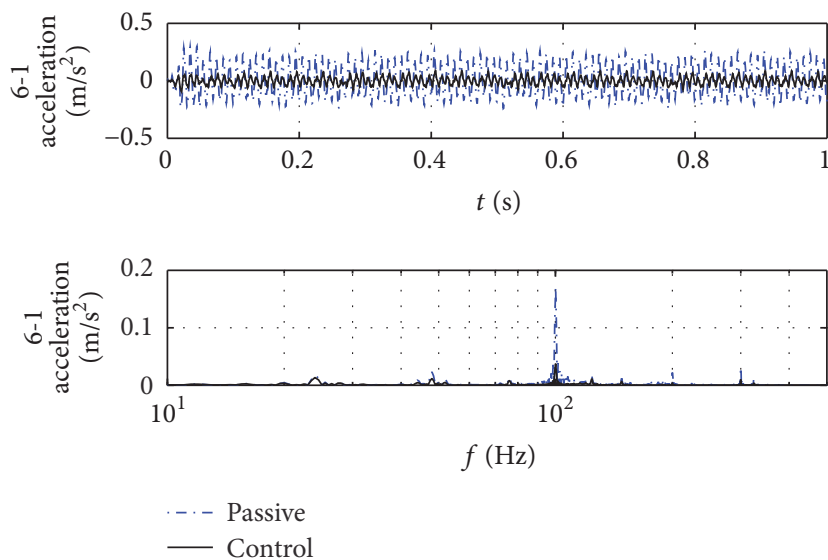

FIGURE 23: Comparison time-frequency domain curves of acceleration response of position 6-1 on the experimental platform before and after being controlled.
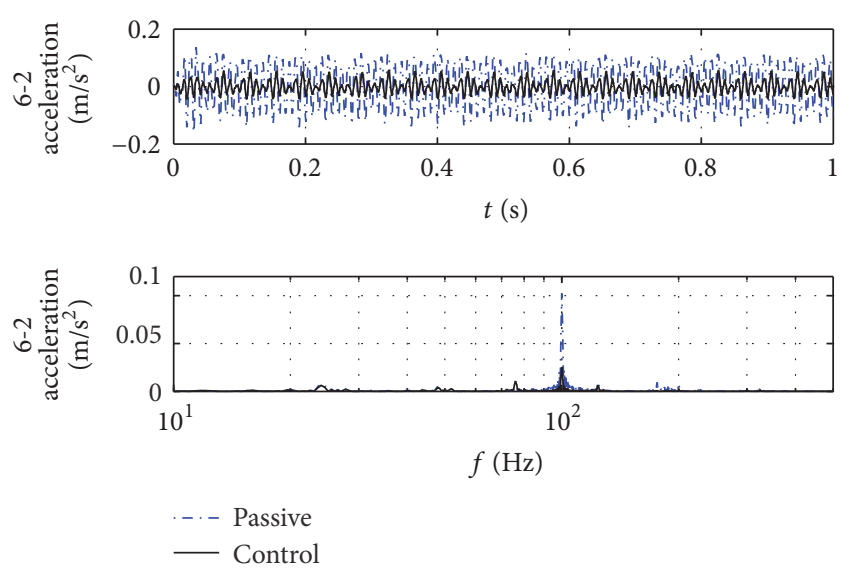

FIgURE 24: Comparison time-frequency domain curves of acceleration response of position 6-2 on the experimental platform before and after being controlled.

active isolation system is only $35 \%$ of the passive system. In the frequency domain curves, the vertical axis represents the magnitude of the acceleration response, and the horizontal axis is time. Likewise, the dotted line represents the response curve of acceleration before being controlled, and the solid line is the response curve of acceleration after being controlled. It can be discovered that the maximum peak amplitude (at $100 \mathrm{~Hz}$ ) of acceleration response reduces from $0.1715 \mathrm{~m} / \mathrm{s}^{2}$ before being controlled to $0.03744 \mathrm{~m} / \mathrm{s}^{2}$ after being controlled, which means it is reduced by $78 \%$. The decrease of other peak amplitudes is also quite significant; the increase of isolation effect is obvious.

The time-frequency domain curves of the output acceleration of position 6-2 in the isolation experimental platform before and after being controlled are shown in Figure 24. In the time domain curves, the dotted line represents the response curve of acceleration before being controlled, and the solid line is the response curve of acceleration after being controlled. It can be seen that the maximum peak-to-peak amplitude of acceleration response is reduced from passive system's $0.2953 \mathrm{~m} / \mathrm{s}^{2}$ before being controlled to active system's
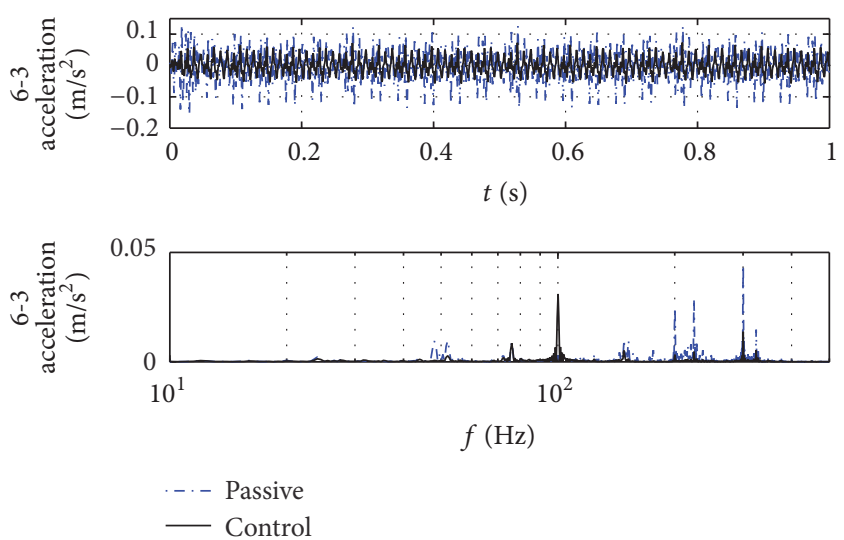

FIGURE 25: Comparison time-frequency domain curves of acceleration response of position 6-3 on the experimental platform before and after being controlled.
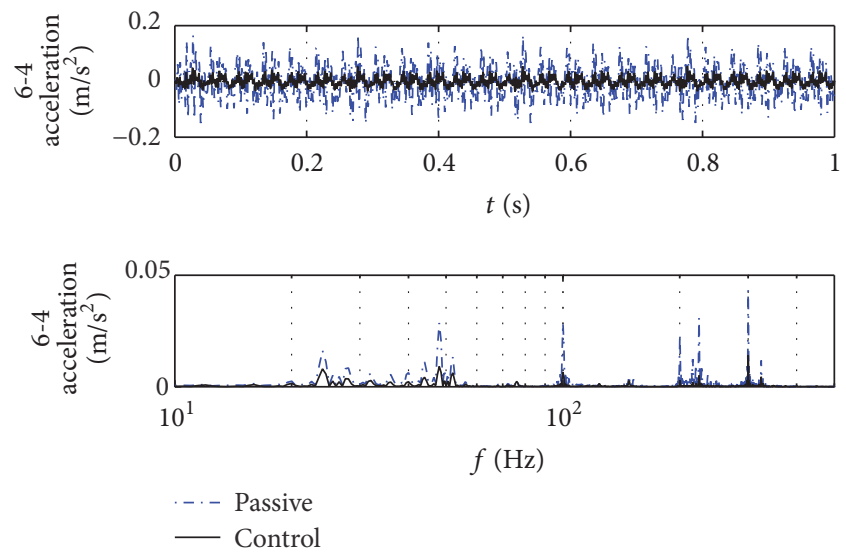

FIGURE 26: Comparison time-frequency domain curves of acceleration response of position 6-4 on the experimental platform before and after being controlled.

$0.1019 \mathrm{~m} / \mathrm{s}^{2}$ after being controlled. The maximum acceleration response of the active isolation system is only around $1 / 3$ of the passive system. In the frequency domain curves, the dotted line represents the response curve of acceleration before being controlled, and the solid line is the response curve of acceleration after being controlled. It can be discovered that the maximum peak amplitude (at $100 \mathrm{~Hz}$ ) of acceleration response is reduced from $0.1021 \mathrm{~m} / \mathrm{s}^{2}$ before being controlled to $0.02548 \mathrm{~m} / \mathrm{s}^{2}$ after being controlled, which means it is reduced by $75 \%$. The increase of isolation effect is obvious.

The time-frequency domain curves of the output acceleration of position 6-3 in the isolation experimental platform before and after being controlled are shown in Figure 25. In the time domain curves, the dotted line represents the response curve of acceleration before being controlled, and the solid line is the response curve of acceleration after being controlled. It can be seen that the maximum peakto-peak amplitude of acceleration response is reduced from passive system's $0.2865 \mathrm{~m} / \mathrm{s}^{2}$ before being controlled to active system's $0.13078 \mathrm{~m} / \mathrm{s}^{2}$ after being controlled. The maximum acceleration response of the active isolation system is only 
around $45 \%$ of the passive system. In the frequency domain curves, the dotted line represents the response curve of acceleration before being controlled, and the solid line is the response curve of acceleration after being controlled. It can be discovered that the maximum peak amplitude (at $200 \mathrm{~Hz}$ ) of acceleration response is reduced from $0.04424 \mathrm{~m} / \mathrm{s}^{2}$ before being controlled to $0.03103 \mathrm{~m} / \mathrm{s}^{2}$ after being controlled, which means it is reduced by $25 \%$. The decrease of other peak amplitudes is also quite significant.

The time-frequency domain curves of the output acceleration of position 6-4 in the isolation experimental platform before and after being controlled are shown in Figure 26. In the time domain curves, the dotted line represents the response curve of acceleration before being controlled, and the solid line is the response curve of acceleration after being controlled. It can be seen that the maximum peakto-peak amplitude of acceleration response is reduced from passive system's $0.3188 \mathrm{~m} / \mathrm{s}^{2}$ before being controlled to active system's $0.09127 \mathrm{~m} / \mathrm{s}^{2}$ after being controlled. The maximum acceleration response of the active isolation system is only around $28 \%$ of the passive system. In the frequency domain curves, the dotted line represents the response curve of acceleration before being controlled, and the solid line is the response curve of acceleration after being controlled. It can be discovered that the maximum peak amplitude (at $200 \mathrm{~Hz}$ ) of acceleration response is reduced from $0.04336 \mathrm{~m} / \mathrm{s}^{2}$ before being controlled to $0.01407 \mathrm{~m} / \mathrm{s}^{2}$ after being controlled, which means it is reduced by more than $68 \%$. The decrease of other peak amplitudes is also quite significant.

\section{Conclusions}

Multisources excitation active vibration isolation system has complex structure and strong coupling ability. For those features, the model identification method is adopted to establish active isolation system model, and the model identification accuracy is over $90 \%$. PID control model based on acceleration feedback is established, and an improved PSO algorithm of a dynamic inertia weight factor is put forward. Optimization for established PID control parameters is conducted. In order to verify the effectiveness of control model and the control parameters obtained by improved PSO algorithm, simulation analysis is carried out under the functions of double sine interference signals and double sweep-frequency signals. The simulation results show that the vibration acceleration amplitudes of active multisources excitation isolation system after being controlled are much smaller than the passive system before being controlled, and the isolation effect of the active system is much better than those of the passive system. In the end, experimental verification is carried out. The vibration isolation effect of the active control of multisources excitation isolation system agrees with the simulation results. The maximum output acceleration amplitude of the active system is only $40 \%$ of the passive system, and the vibration isolation effect is significantly improved.

\section{Competing Interests}

The authors declare that they have no competing interests.

\section{Acknowledgments}

The authors would like to acknowledge the National Natural Science Foundation of China (no. 51205296, no. 51275368, and no. U1537103).

\section{References}

[1] T. Yang, D. Huang, L. Zhou et al., "Experimental investigation of an active vibration isolation system for a floating raft based on a flexible hull-like structure," in Proceedings of the 21st International Congress on Sound and Vibration (ICSV21 '14), pp. 14-17, Beijing, China, July 2014.

[2] H. L. Sun, K. Zhang, P. Q. Zhang, and H. B. Chen, "Application of dynamic vibration absorbers in floating raft system," Applied Acoustics, vol. 71, no. 3, pp. 250-257, 2010.

[3] T. Yang, Y. Sun, L. Zhou, M. J. Brennan, and Z. Liu, "Practical demonstration of a large-scale active vibration isolation system," Case Studies in Mechanical Systems and Signal Processing, vol. 1, pp. 32-37, 2015.

[4] J. Orivuori, I. Zazas, and S. Daley, "Active control of frequency varying disturbances in a diesel engine," Control Engineering Practice, vol. 20, no. 11, pp. 1206-1219, 2012.

[5] Y. Yun and Y. Li, "A general dynamics and control model of a class of multi-DOF manipulators for active vibration control," Mechanism and Machine Theory, vol. 46, no. 10, pp. 1549-1574, 2011.

[6] W. Hamblen, "Next generation stealth submarines," Sea Technology, vol. 39, no. 11, pp. 59-62, 1998.

[7] K. Park, D. Choi, A. Ozer, S. Kim, Y. Lee, and D. Joo, "A voice coil actuator driven active vibration isolation system with the consideration of flexible modes," Review of Scientific Instruments, vol. 79, no. 6, Article ID 065106, 2008.

[8] S. Daley, F. A. Johnson, J. B. Pearson, and R. Dixon, "Active vibration control for marine applications," Control Engineering Practice, vol. 12, no. 4, pp. 465-474, 2004.

[9] S. Daley, F. A. Johnson, J. B. Pearson, R. Harrison, F. Zhang, and I. Zazas, "Active control of vibration in marine systems," in Proceedings of the UKACC Control Mini Symposia, pp. 191-200, IET, Glasgow, UK, August 2006.

[10] Y. Liu, H. Matsuhisa, and H. Utsuno, "Semi-active vibration isolation system with variable stiffness and damping control," Journal of Sound and Vibration, vol. 313, no. 1-2, pp. 16-28, 2008.

[11] T. J. Yang, G. Y. Jin, W. Y. Li, Z. G. Liu, W. P. Zhang, and Z. Q. Wang, "Study on active control techniques for warship power plant," Ship Science and Technology, vol. 28, supplement 2, pp. 47-51, 2006.

[12] C. Zhao and D. Chen, "Semi-active fuzzy sliding mode control for floating raft isolation system," Chinese Journal of Mechanical Engineering, vol. 44, no. 2, pp. 163-169, 2008.

[13] M. E. Hoque, M. Takasaki, Y. Ishino, H. Suzuki, and T. Mizuno, "An active micro vibration isolator with zero-power controlled magnetic suspension technology," JSME International Journal, Series C, vol. 49, no. 3, pp. 719-726, 2006.

[14] M. A. Swinbanks and S. Daley, Advanced Submarine Technology-project M Control Theory Report, Office of Naval Research, 1993.

[15] F. A. Johnson, Advanced Submarine Technology-project M Control Experiments and Simulations, Office of Naval Research, 1994. 
[16] C.-S. Song, Y. Hu, S. Xie, and Z. Zhou, "Dynamic modeling of magnetic suspension isolator using artificial neural network: a modified genetic approach," Journal of Vibration and Control, vol. 19, no. 6, pp. 847-856, 2013.

[17] C. Song, Z. Zhou, S. Xie, Y. Hu, J. Zhang, and H. Wu, "Fuzzy control of a semi-active multiple degree-of-freedom vibration isolation system," Journal of Vibration and Control, vol. 21, no. 8, pp. 1608-1621, 2015.

[18] X. Chen and Y. Li, "A modified PSO structure resulting in high exploration ability with convergence guaranteed," IEEE Transactions on Systems, Man, and Cybernetics, Part B: Cybernetics, vol. 37, no. 5, pp. 1271-1289, 2007.

[19] Y.-L. Li, W. Shao, L. You, and B.-Z. Wang, "An improved PSO algorithm and its application to UWB antenna design," IEEE Antennas and Wireless Propagation Letters, vol. 12, pp. 12361239, 2013.

[20] N.-J. Li, W.-J. Wang, and C.-C. J. Hsu, "Hybrid particle swarm optimization incorporating fuzzy reasoning and weighted particle," Neurocomputing, vol. 167, pp. 488-501, 2015.

[21] M. Baskin and B. Caglar, "A modified design of PID controller for permanent magnet synchronous motor drives using particle swarm optimization," in Proceedings of the 16th International Power Electronics and Motion Control Conference and Exposition (PEMC '14), pp. 388-393, IEEE, Antalya, Turkey, September 2014.

[22] Y. Lu, D. Yan, J. Zhang, and D. Levy, "A variant with a time varying PID controller of particle swarm optimizers," Information Sciences, vol. 297, pp. 21-49, 2015.

[23] S. Panda, B. K. Sahu, and P. K. Mohanty, "Design and performance analysis of PID controller for an automatic voltage regulator system using simplified particle swarm optimization," Journal of the Franklin Institute, vol. 349, no. 8, pp. 2609-2625, 2012.

[24] A. Moharam, M. A. El-Hosseini, and H. A. Ali, "Design of optimal PID controller using hybrid differential evolution and particle swarm optimization with an aging leader and challengers," Applied Soft Computing Journal, vol. 38, pp. 727737, 2016.

[25] S. M. Gharghory and H. A. Kamal, "Optimal tuning of PID controller using adaptive hybrid particle swarm optimization algorithm," International Journal of Computers Communications \& Control, vol. 7, no. 1, pp. 101-114, 2012.

[26] W.-D. Chang and C.-Y. Chen, "PID controller design for MIMO processes using improved particle swarm optimization," Circuits, Systems, and Signal Processing, vol. 33, no. 5, pp. 14731490, 2014.

[27] T.-H. Kim, I. Maruta, and T. Sugie, "Robust PID controller tuning based on the constrained particle swarm optimization," Automatica, vol. 44, no. 4, pp. 1104-1110, 2008. 


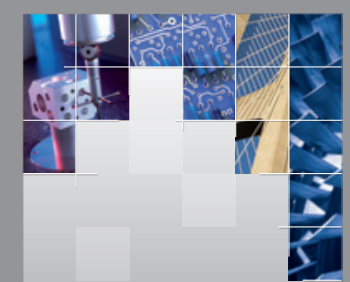

\section{Enfincering}
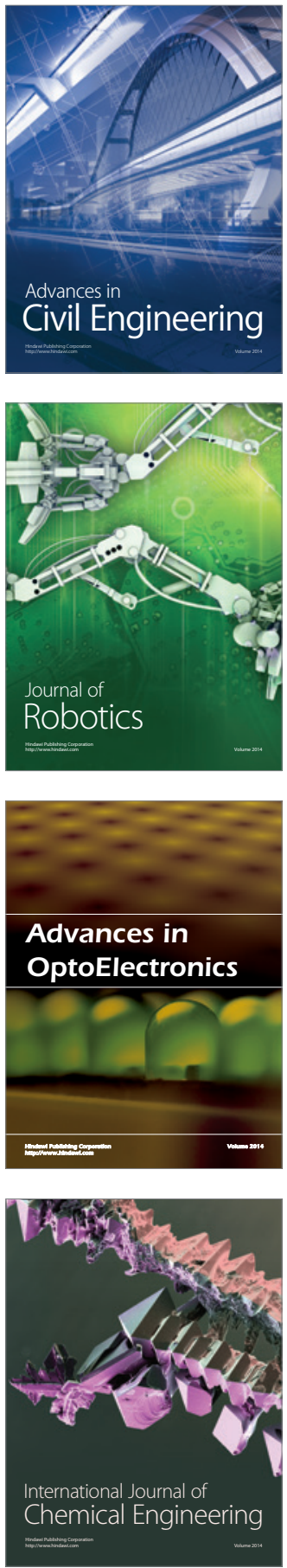

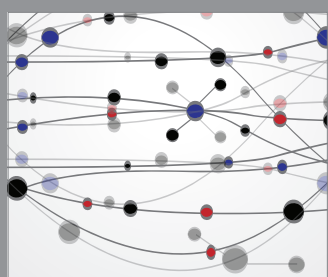

The Scientific World Journal

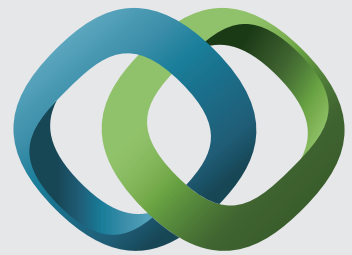

\section{Hindawi}

Submit your manuscripts at

http://www.hindawi.com
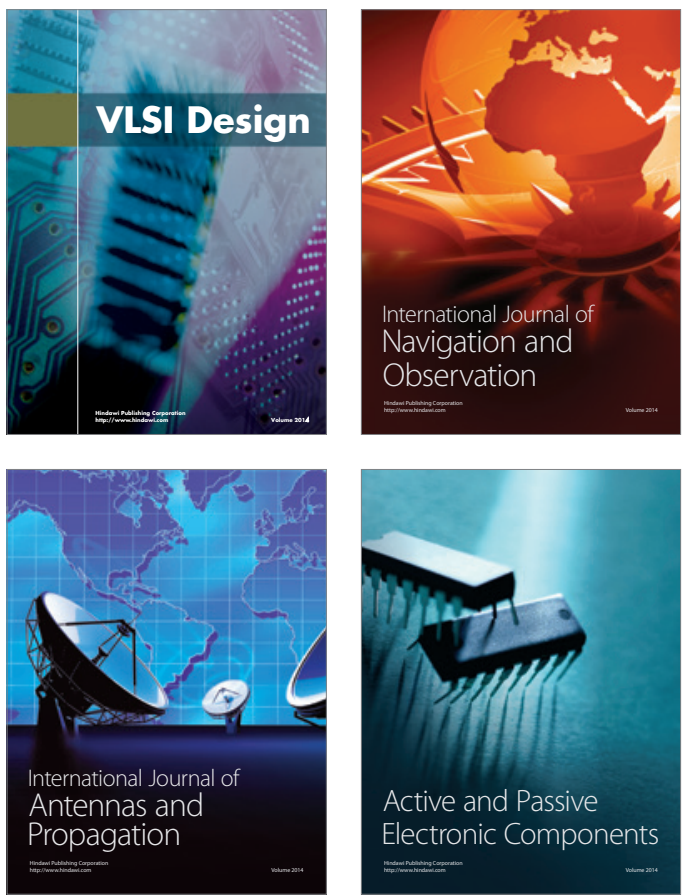
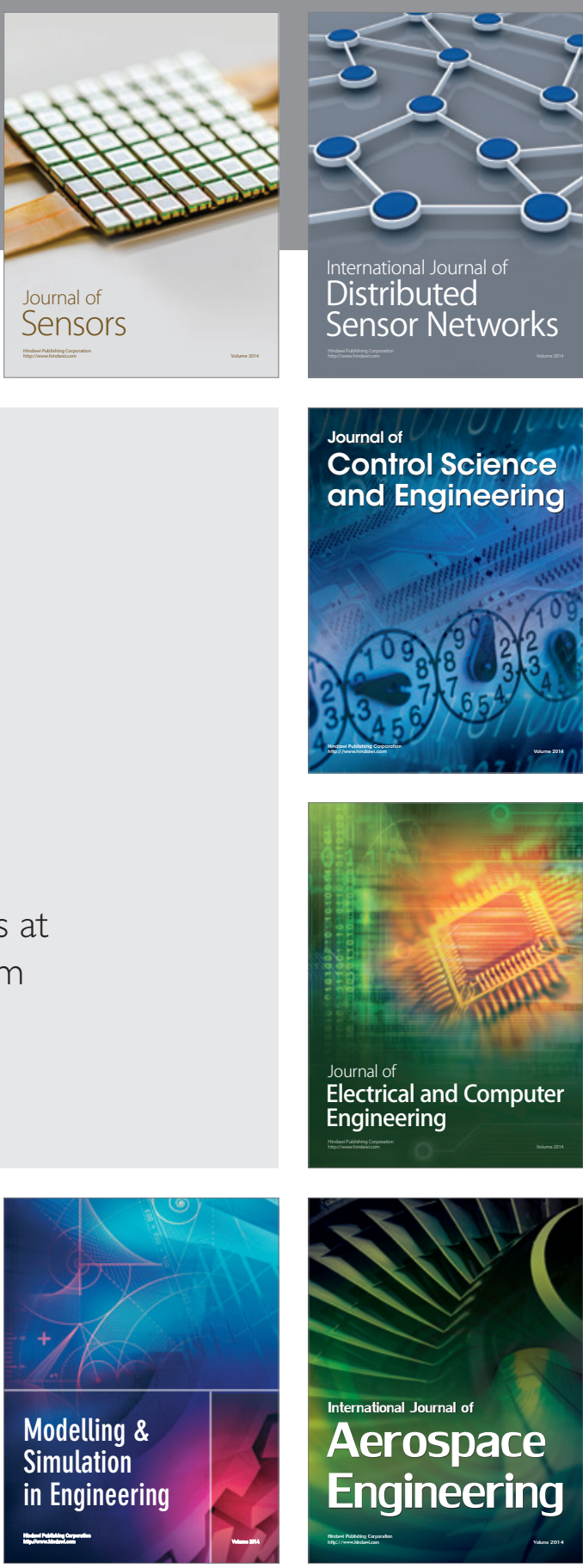

International Journal of

Distributed

Sensor Networks

Journal of

Control Science

and Engineering
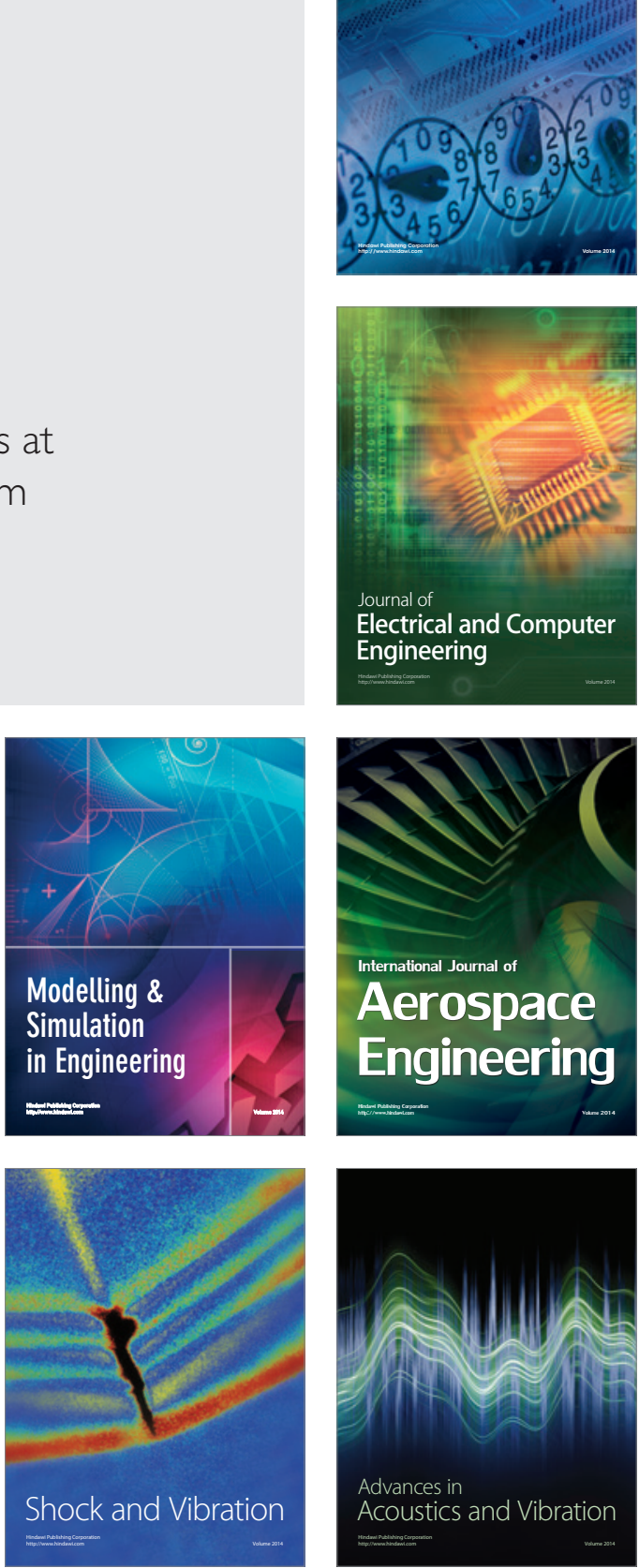